\title{
Megahertz-compatible angular streaking with few-femtosecond resolution at x-ray free-electron lasers
}

\author{
R. Heider, ${ }^{1}$ M. S. Wagner, ${ }^{1}$ N. Hartmann,,${ }^{2,3,4}$ M. Ilchen, ${ }^{5,6,7}$ J. Buck, ${ }^{5,8}$ G. Hartmann, ${ }^{7,9}$ V. Shirvanyan, ${ }^{1}$ A. O. Lindahl, ${ }^{2,10,11}$ \\ J. Grünert, ${ }^{5}$ J. Krzywinski, ${ }^{2}$ J. Liu, ${ }^{5}$ M. Ossiander, ${ }^{1,12}$ A. A. Lutman $\odot,{ }^{2}$ T. Maxwell, ${ }^{2}$ A. A. Miahnahri, ${ }^{2}$ S. P. Moeller, ${ }^{2}$ \\ M. Planas, ${ }^{5}$ J. Robinson, ${ }^{2}$ J. Viefhaus,,${ }^{9, *}$ T. Feurer ${ }^{3}$ R. Kienberger, ${ }^{1,12}$ R. N. Coffee,,${ }^{2,6}$ and W. Helml ${ }^{1,13,14, \uparrow}$ \\ ${ }^{1}$ Technische Universität München, 85748 Garching, Germany \\ ${ }^{2}$ SLAC National Accelerator Laboratory, Linac Coherent Light Source, Menlo Park, California 94025, USA \\ ${ }^{3}$ University of Bern, Institute of Applied Physics, 3012 Bern, Switzerland \\ ${ }^{4}$ Coherent Inc., 5100 Patrick Henry Drive, Santa Clara, California 95054, USA \\ ${ }^{5}$ European XFEL GmbH, Holzkoppel 4, 22869 Schenefeld, Germany \\ ${ }^{6}$ PULSE Institute, Stanford University and SLAC National Accelerator Laboratory, Menlo Park, California 94025, USA \\ ${ }^{7}$ Institut für Physik und CINSaT, Universität Kassel, Heinrich-Plett-Straße 40, 34132 Kassel, Germany \\ ${ }^{8}$ Institut für Experimentelle und Angewandte Physik, Christian-Albrechts-Universität zu Kiel, Leibnizstraße 11-19, 24098 Kiel, Germany \\ ${ }^{9}$ Deutsches Elektronen-Synchrotron DESY, 22607 Hamburg, Germany \\ ${ }^{10}$ University of Gothenburg, Department of Physics, SE-412 96 Gothenburg, Sweden \\ ${ }^{11}$ Qamcom Research \& Technology AB, Falkenbergsgatan 3, SE-412 85 Göteborg, Sweden \\ ${ }^{12}$ Max-Planck-Institut für Quantenoptik, 85748 Garching, Germany \\ ${ }^{13}$ Ludwig-Maximilians-Universität München, 85748 Garching, Germany \\ ${ }^{14}$ Zentrum für Synchrotronstrahlung (DELTA), Technische Universität Dortmund, 44227 Dortmund, Germany
}

(Received 6 March 2019; revised manuscript received 4 September 2019; published 25 November 2019)

\begin{abstract}
Highly brilliant, coherent, femtosecond x-ray pulses delivered by free-electron lasers (FELs) constitute one of the pillars of modern ultrafast science. Next generation FEL facilities provide up to megahertz repetition rates and pulse durations down to the attosecond regime utilizing self-amplification of spontaneous emission. However, the stochastic nature of this generation mechanism demands single-shot pulse characterization to perform meaningful experiments. Here we demonstrate a fast yet robust online analysis technique capable of megahertz-rate mapping of the temporal intensity structure and arrival time of $\mathrm{x}$-ray FEL pulses with few-femtosecond resolution. We performed angular streaking measurements of both neon photo- and Auger electrons and show their applicability for a direct time-domain feedback system during ongoing experiments. The fidelity of the real-time pulse characterization algorithm is corroborated by resolving isolated x-ray pulses and double pulse trains with few-femtosecond substructure, thus paving the way for x-ray-pump-x-ray-probe FEL science at repetition rates compatible with the demands of LCLS-II and European XFEL.
\end{abstract}

DOI: 10.1103/PhysRevA.100.053420

\section{INTRODUCTION}

Modern high-brightness free-electron lasers (FELs) with a wide range of tunable photon energies up to the hard $\mathrm{x}$-ray regime and pulse durations on the femtosecond timescale offer the possibility of time-resolved studies addressing a large variety of element-specific and chemical-state-selective processes [1-3]. Therefore, x-ray pump-probe experiments have become a powerful tool for time-resolved atomic and molecular physics [4-6], biology [7], chemistry [8], and materials science [9]. As the time resolution of these studies is already approaching the attosecond regime [10-12], a reliable and noninvasive technique for directly measuring

*Present address: Helmholtz-Zentrum Berlin fr Materialien und Energie, Albert-Einstein-Strasse 15, 12489 Berlin, Germany.

†Corresponding author: wolfram.helml@tu-dortmund.de, Zentrum für Synchrotronstrahlung (DELTA), Technische Universität Dortmund, 44227 Dortmund, Germany. the temporal structure of these ultrashort FEL pulses is crucial [13]. Especially at machines using self-amplification of spontaneous emission (SASE) this has been a long-standing diagnostic goal [14]. Indirect methods, like the pulse duration measurement based on electron energy losses in the undulator using a transverse deflection cavity (XTCAV) [15], often fail to give relevant information on the pulse structure, due to slippage effects [16] or because in novel methods of FEL generation different parts of the electron bunch are used to build up the same single x-ray pulse [17] or multiple pulses with variable delay [18]. Photoelectron streaking with fewcycle, carrier-envelope-stabilized, mid-infrared (MIR) pulses generating isolated attosecond pulses via high harmonic generation $[19,20]$ has revealed electron dynamics in various gaseous [21,22] and solid-state samples [23], but is also an important tool to characterize the electric field of the pulses involved [24]. Streaking with linearly polarized optical or terahertz pulses has already been used for the single-shot determination of an upper length limit of soft x-ray FEL pulses [25] or for the measurement of their durations [26-28] 
in the femtosecond regime. Recently, terahertz streaking was also implemented for time stamping of a relativistic electron beam [29], as used for example in ultrafast electron diffraction measurements. Finally, the concept of angular streaking was applied to unravel the time-energy structure of x-ray pulses using an iterative retrieval algorithm [30]. Nevertheless, this full reconstruction is only suitable for pulse sorting after the experiment due to its high computational demands.

Here we demonstrate the single-shot pulse characterization of the temporal intensity structure of $\mathrm{x}$-ray pulses with few-femtosecond resolution based on a fast algorithm, which enables online data processing at modern XFEL facilities operating at up to $\mathrm{MHz}$ repetition rates [31,32]. The approach is simple, straightforward, and intuitive in view of the classical concept of angular streaking. The algorithm is based on an angle-dependent measurement of the circularly streaked photoelectron intensity distribution [33,34]. This angle-dependent signal is mapped to a representation of the temporal intensity profile of the $\mathrm{x}$-ray pulse via the attoclock principle $[35,36]$. Either photoelectrons or Auger electrons from a dilute gas target can be used to measure the profile of $x$-ray SASE FEL pulses of different durations with negligible $x$-ray beam intensity losses and independent from simultaneously ongoing experiments with a resolution of about 3.5 fs. In addition, we validate this fast approach by comparing retrieved pulse structures with the results of the recently published attosecond time-energy reconstruction [30] mentioned above.

The typical substructure of an $\mathrm{x}$-ray FEL pulse operated on the SASE principle is complicated due to shot-to-shot fluctuations of the electron accelerator and the stochastic nature of the generation process [37]. Due to the exponential amplification of spontaneous emission in the undulator, subsections of the electron bunch have no fixed phase relation and act as essentially independent radiation sources [14]. This yields a train of multiple SASE spikes, each stemming from a coherent emission process, with random carrier-envelope phase jumps in between and a temporal intensity substructure varying from shot to shot $[38,39]$.

The principle of photoelectron streaking as a measurement of the momentum change of extreme ultraviolet (XUV)- or $\mathrm{x}$ ray-generated electrons caused by a linearly polarized optical laser $[24,40]$ can be applied to x-ray FEL pulses. However, an accurate time overlap with a certain phase of the streaking field is not guaranteed due to a significant arrival time jitter between the optical and $\mathrm{x}$-ray pulses [41-43]. Therefore, up to now linear streaking experiments are not capable of resolving the temporal substructure of FEL pulses based on the principle of self-amplification of spontaneous emission. Angular streaking addresses this issue using a circularly polarized streaking laser and an angle-dependent detection method for the photoelectrons (see Appendix A). Since the magnitude of the streaking field is nearly constant within one rotation period a particular phase relation of the x-ray pulse with respect to the streaking field is not required. Thus, the photoelectrons are accelerated in different radial directions by the rotating electric field of the streaking laser, depending on the instant of ionization [35]. The temporal intensity structure of the x-ray pulse can be retrieved, as it is mapped to angle-dependent energy shifts of photoelectron spectra [36].

\section{EXPERIMENT}

At the Atomic, Molecular, and Optical Science (AMO) end station of the Linac Coherent Light Source (LCLS), we conducted ionization experiments of a dilute neon gas target. The x-ray pulses had estimated average root-mean-squared (rms) pulse durations of 3.5, 6.5, and 10.5 fs [44] at a photon energy of $1180 \mathrm{eV}$ (see Appendix B). Hereby, electrons stemming from two different generation processes have been deployed as measuring probes: Ne $1 s$ core electrons $\left(E_{\text {bind }}=\right.$ $870 \mathrm{eV}, E_{\text {kin }}=310 \mathrm{eV}$ ) as well as $K-L L$ Auger electrons $\left(\bar{E}_{\mathrm{kin}}^{\mathrm{Aug}} \approx 803.5 \mathrm{eV}\right)[45]$. They were streaked angularly by a MIR laser with $\lambda=10.6 \mu \mathrm{m}$ (see Appendix C), resulting in a 35.3 fs rotation period of the electric field vector $[30,35]$. The time- and angle-dependent electron yield is measured by a circular assembly of 16 individually operated timeof-flight detectors, evenly distributed around the interaction region in steps of $22.5^{\circ}$ in the plane perpendicular to the x-ray propagation axis (see Appendices D and E) [46,47]. The FEL pulses are focused in a dilute neon gas target (interaction region) centered in the acceptance volume of the spectrometer array. The energy resolution of a single timeof-flight detector is experimentally determined to lie between 0.75 to $1 \mathrm{eV}$.

The main principle of an angular streaking experiment can be clarified by the comparison of the angle-dependent spectral photoelectron distributions $S(E, \varphi)$ for the unstreaked and streaked cases in Fig. 1, where $E$ is the kinetic energy and $\varphi$ is the detection angle. The angle-dependent photoelectron energy shift due to the orientation of the streaking field vector at the instant of ionization is clearly visible. For a specific detector, the spectrum experiences a shift to higher energies only if the electric field vector of the MIR streaking field is pointing in the angular direction of the detector during the presence of the x-ray pulse. The highest photoelectron energy recorded in each detector [cutoff energy $E_{\mathrm{cut}}(\varphi)$ ] serves as a single-shot measure for the relative temporal overlap between the X-ray and MIR pulses, and is determined by the respective energy value where the signal intensity drops below $10 \%$ of its maximum value [see Figs. 1(c) and 1(d)].

\section{THE INTEGRATION PULSE CHARACTERIZATION ALGORITHM}

\section{A. Determination of the MIR elliptical polarization}

Due to the elliptical, thus noncircular polarization of the infrared streaking laser, its amplitude shows a sinusoidal characteristic depending on the direction of the streaking field vector potential in the polarization plane. Consequently, the extent of the shift of the electron energy distribution to lower or higher energies induced by the streaking field is inherently dependent on the angular position of the respective detector. Thus, it is indispensable to consider these differences in the maximum potential streaking shift for every flight tube and to correct this deviation from a perfectly circularly polarized streaking field.

For this purpose, the polarization ellipse is determined in the following way: First, for each detector and each FEL shot, the maximum streaking shift to higher energies is identified by the energy value in the spectrum where the 
(a)

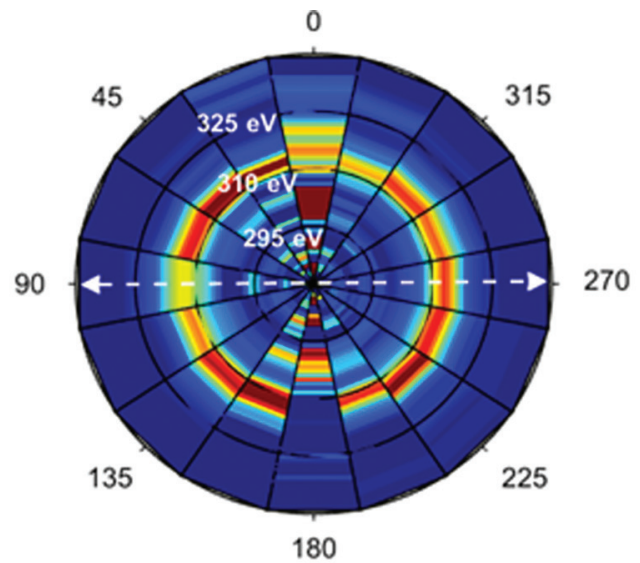

(c)

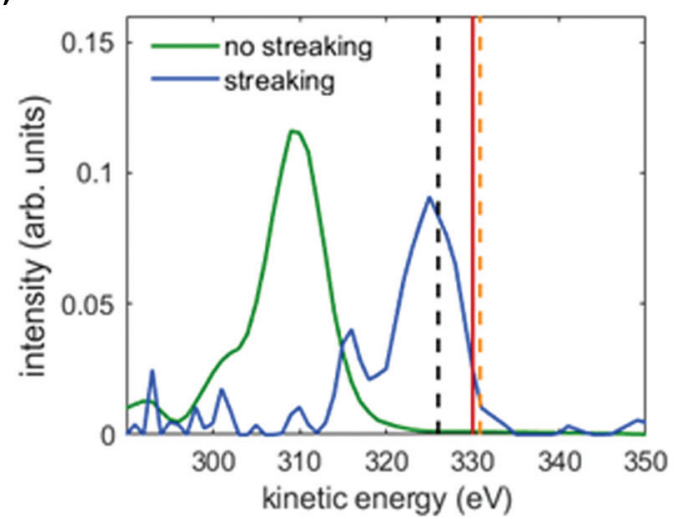

(b)

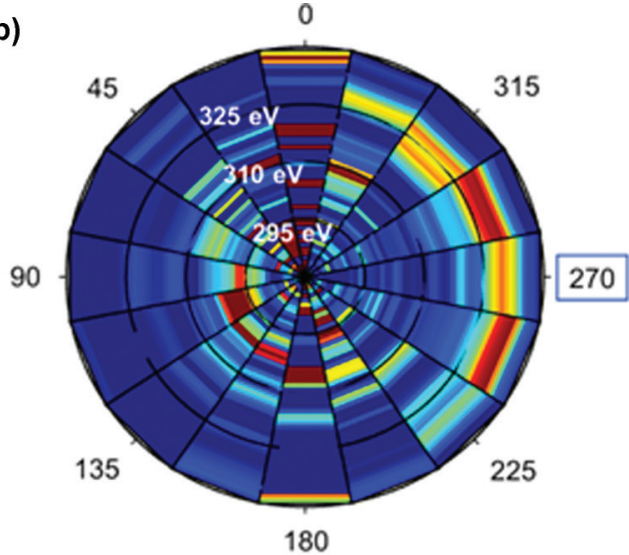

(d)

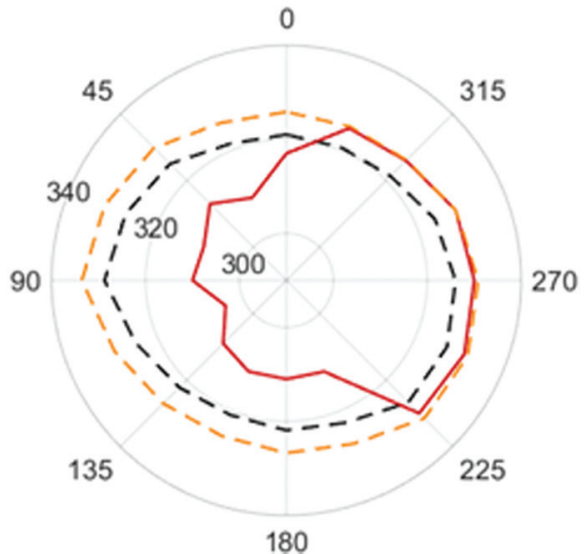

FIG. 1. Visualization of the effect of angular steaking based on experimental data in the spectral domain. The upper panels show singleshot, normalized, and energy-calibrated Ne $1 s$ photoelectron spectrogramlike polar images $S(E, \varphi)$ recorded by the e-TOF spectrometer array in the plane perpendicular to the FEL beam propagation direction in the absence (a) and in the presence (b) of the MIR streaking laser field. In the nonstreaking situation (a), the kinetic energy of the Ne $1 s$ photoelectrons excited by an FEL photon energy of $1180 \mathrm{eV}$ is constant over all angles, whereas the close-to-circularly polarized streaking field (b) modulates the spectra depending on the $\mathrm{x}$-ray pulse substructure and the detection angle, according to the principle of angular streaking. The horizontal linear polarization direction of the FEL beam is indicated by the white, dashed arrow in (a). (c) The streaked spectrum recorded by the detector at $270^{\circ}$ [blue line, also highlighted in (b)] is shown in comparison to an unstreaked spectrum (green line). The energy values $E_{\text {low }}$ (vertical black dashed line), $E_{\text {cut }}$ (vertical red full line), and $E_{\text {up }}$ (vertical yellow dashed line), relevant for the integration pulse retrieval algorithm, are marked for this specific shot and detector. In (d), these angle-dependent energy values are shown in a polar plot, where the maximum energy is observed at $292.5^{\circ}$.

signal intensity drops below $10 \%$ of its maximum value, representing the single-shot cutoff energy $E_{\text {cut }}(\varphi)$. Then we set the overall streaking limit $E_{\text {ell }}(\varphi)$ of each detector to the energy value where the respective distribution of these cutoff energies $E_{\text {cut }}(\varphi)$ over all shots has dropped below $2 \%$ of its maximum on the high-energy side of the distribution. This ensures that the actual maximum streaking shift is independently determined for each detector, while mostly eliminating the influence of shot noise. All shots where the cutoff energy $E_{\text {ell }}(\varphi)$ is detected to be apparently higher than this overall streaking $\operatorname{limit} E_{\mathrm{ell}}(\varphi)$ for at least one detector are discarded. Eventually, orientation, shape, and size of the polarization ellipse of the MIR streaking field are reflected by the resulting, detector-related energy values for the overall streaking limit, as shown in Fig. 2 for the analyzed data sets. For the further analysis, a normalized polarization ellipse $\mathcal{E}_{\text {norm }}(\varphi)$ is calculated by dividing the angledependent, overall streaking limits $E_{\text {ell }}(\varphi)$ by their maximum value.

\section{B. Correction of the measured cutoff energy for the elliptical polarization of the MIR laser}

Subsequently, the cutoff energy $E_{\text {cut }}(\varphi)$ measured by each detector for every FEL shot can now be corrected for the elliptical polarization of the MIR laser on a single-shot basis. First, the angle-dependent, maximum energy shift to higher energies caused by the streaking field in each TOF detector is determined to

$$
E_{\text {shift }}(\varphi)=\frac{E_{\text {cut }}(\varphi)-E_{0}}{\mathcal{E}_{\text {norm }}(\varphi)},
$$

where $E_{\text {cut }}(\varphi)$ is the single-shot cutoff energy, $\mathcal{E}_{\text {norm }}(\varphi)$ is the normalized polarization ellipse, and $E_{0}$ is the unstreaked energy of the Ne $1 s$ photoline or the main Auger line, respectively. Then, the ellipse-corrected, maximum energy shift of all flight tubes $a_{\mathrm{ell}}$ for the respective shot is ascertained to

$$
a_{\mathrm{ell}}=\max \left[E_{\text {shift }}(\varphi)\right]
$$


Polarization Ellipse Neon Auger data sets 0

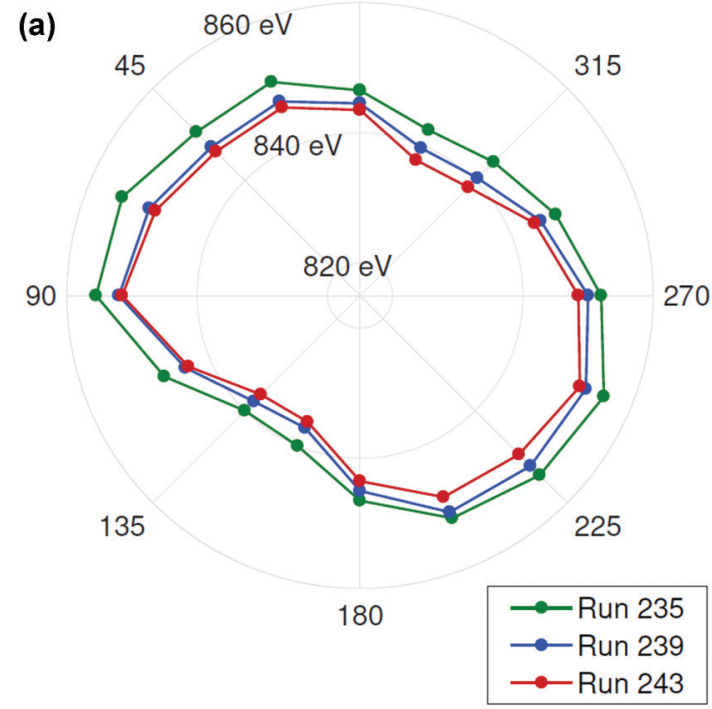

Polarization Ellipse Neon 1s data sets

0

(b)
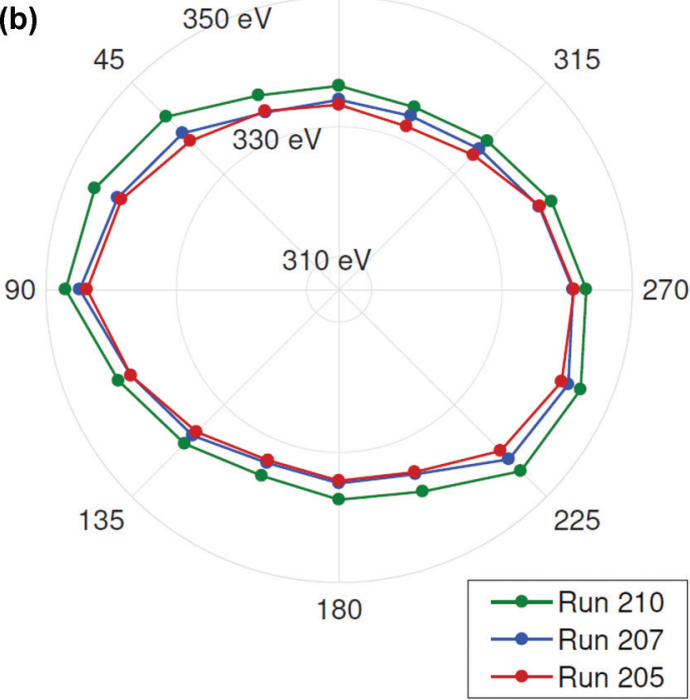

FIG. 2. Illustration of the ascertained polarization ellipse. The polarization ellipse illustrated by the angle-dependent overall streaking limit $E_{\text {ell }}(\varphi)$ is depicted in a polar plot for (a) the three Auger electron and (b) the three photoelectron data sets, respectively. The slight deviation from an elliptical shape in (a) might be introduced by a small nonlinearity for the high retardation voltage settings (770 V) used in these measurements for the streaking of the high-energy Ne $K-L L$ Auger electrons.

The scaling factor $a_{\mathrm{ell}}$, the so-called streaking kick, varies from shot to shot, as the actual amplitude of the streaking field envelope depends on the relative $\mathrm{x}$-ray-optical arrival time. Eventually, by multiplying the current streaking kick as a scaling factor and adding the unstreaked energy $E_{0}$ again, one obtains an (ellipse-corrected), single-shot upper energy limit for the shifted electron signal in each detector:

$$
E_{\text {up }}(\varphi)=a_{\text {ell }} \mathcal{E}_{\text {norm }}(\varphi)+E_{0} .
$$

The values of $E_{\mathrm{up}}(\varphi)$ for each detector shape a single-shot, streaking kick ellipse that indicates the maximal possible electron energy that could be detected by the respective detector with respect to the current magnitude of the streaking kick, if the streaking vector potential pointed in the direction of this detector at the instant of ionization.

\section{Characterization of the temporal structure of the $x$-ray pulse by spectral integration}

The $\mathrm{x}$-ray intensity at a given time is directly proportional to the number of generated electrons [48], corrected for the detector transmission and for the different emission characteristics of $\mathrm{Ne} 1 s$ and Auger electrons. For eventually obtaining a meaningful measure of the x-ray pulse shape, the spectral intensity signal of each individual spectrometer is integrated over an energy range below the corresponding cutoff energy as theoretically derived and discussed in [34]. Below its cutoff energy range, a detector at a given angular position additionally records to some degree energy-shifted electrons from projections of the streaking vector potential, pointing at angles close to the direction of the detector under consideration during the presence of the ionizing $\mathrm{x}$-ray pulse. Thus, we choose a carefully selected narrow integration window for achieving the highest possible temporal resolution regarding the retrieval of the intensity structure of the x-ray pulses.

By our definitions (see above), the single-shot values of $E_{\text {up }}(\varphi)$ given by the streaking kick ellipse constitute an upper limit of the streaking shift for each detector. For the determination of the lower bound for the integral

$$
E_{\text {low }}(\varphi)=E_{\text {up }}(\varphi)-w_{\text {int }},
$$

an integration window

$$
w_{\text {int }}=\left[1-\cos \left(\frac{\pi}{5}\right)\right] a_{\mathrm{ell}}
$$

must be defined, which is adjusted from shot to shot depending on the actual streaking kick $a_{\text {ell }}$. By doing this, one ensures that always the same, relative number of photoelectrons is integrated for each FEL shot, independent of the streaking kick. For a constant integration width, a small streaking kick would imply that a much more electron signal is situated within the integration window with respect to a larger streaking kick, which spreads the electron distribution over a broader energy range. The cosine prefactor sets a finite integration width and thus the lower bound of integration $E_{\mathrm{low}}$, corresponding to a projection angle coverage of $\pm \frac{\pi}{5} \mathrm{rad}$ or $36^{\circ}$ in the polarization plane. This can be interpreted as an angular resolution of roughly one and a half times the angular separation between neighboring detectors in each direction. This integration width represents a compromise between a sufficiently broad, thus well-defined integral and a best possible temporal resolution resulting from a narrow integration window.

Eventually, the spectral photoelectron distribution $S(E, \varphi)$ is integrated over the range determined by the lower integration bound $E_{\text {low }}(\varphi)$ and the cutoff energy $E_{\text {cut }}(\varphi)$ as the upper 
bound. In case that the cutoff energy is situated below the lower bound of integration the integral is set to zero:

$$
\begin{gathered}
I_{\mathrm{x}-\text { ray }}(\varphi)=\int_{E_{\text {low }}(\varphi)}^{E_{\text {cut }}(\varphi)} \mathrm{S}(E, \varphi) d E, \quad E_{\text {cut }}(\varphi)>E_{\text {low }}(\varphi), \\
I_{\mathrm{x}-\text { ray }}(\varphi)=0, \quad E_{\text {cut }}(\varphi) \leqslant E_{\text {low }}(\varphi) .
\end{gathered}
$$

For a specific detector, the integration result is therefore nonzero only if any electrons are energetically up-shifted into the integration window due to the streaking field. Finally, we sort out less reliable reconstructions by discarding those where the summed-up intensity over all detectors is lower than a threshold, empirically defined at $50 \%$ of the maximum value of all shots for that run.

At this point the idea of the "attoclock" (see Appendix A) is applied. The angle-dependent electron intensity $I(\varphi)$ is referred to the angular position of the respective detector and converted into a time dependence of the x-ray pulse intensity with a time axis defined by $t=\frac{\varphi}{2 \pi} \frac{\lambda}{c_{0}}$. For this, one translates the $360^{\circ}$ detection plane into a kind of a chronometer. Using the rotation period of the streaking vector potential as the clock face that amounts to $35.3 \mathrm{fs}$ in our case for a streaking laser wavelength of $\lambda=10.6 \mu \mathrm{m}$. The integration results of all spectrometers can be plotted versus the generated time axis. Thus, one obtains a replica of the $\mathrm{x}$-ray pulse shape in the time regime or, more precisely, a reconstruction of the temporal intensity profile of the $\mathrm{x}$-ray pulse [34]. The pulse duration is determined by calculating the root-mean-squared (rms) widths of the reconstructed intensity profiles instead of a full width at half maximum (FWHM), since the assumption of a Gaussian shape is not necessarily fulfilled for SASE pulses [47]. According to the detector spacing of $22.5^{\circ}$ and the 35.3 fs rotation period of the electric field vector, the best achievable temporal resolution is $2.2 \mathrm{fs}$. However, the actual resolution of the presented integration algorithm is derived to 3.5 fs owing to the smearing of the reconstructed pulse profiles, induced by the streaking field projections from neighboring detectors as discussed above. This value is additionally corroborated by the comparison with the iterative, high-resolution time-energy retrieval [30], which is presented further below.

As a summary, the entire retrieval procedure presented so far is illustrated in Fig. 3 for a single shot from an Auger data set. The single spectra of the independent detectors are presented, where the respective cutoff energy $E_{\text {cut }}$ as well as the upper limit for the streaking shift $E_{\mathrm{up}}$ and the lower integration bound $E_{\text {low }}$ are distinctly marked. Furthermore, a combined depiction of the three characteristic energy values for all detectors in a polar view and the final reconstructed temporal intensity structure of the $\mathrm{x}$-ray pulse are displayed.

\section{RESULTS}

\section{A. Single-shot $\mathrm{x}$-ray pulse characterization}

Polar depictions for three FEL shots of different pulse durations, showing the radially shifted cutoff energy values $E_{\text {cut }}(\varphi)$, the calculated integration window for all detectors and the corresponding temporal intensity profiles of the $\mathrm{x}$-ray pulses are displayed in Fig. 4, together with the retrieved pulse durations. The integration algorithm applied on angular streaking measurements unambiguously resolves variations in the intensity substructure of SASE x-ray pulses and thus determines the pulse duration.

A few tens of thousands of shots for each of the three independent FEL settings, providing estimated averaged pulse durations of 3.5, 6.5, and 10.5 fs have been evaluated, utilizing both $\mathrm{Ne} 1 s$ photoelectrons and Ne Auger electrons as a measuring probe. The resulting histograms of the pulse durations and their mean values derived by the integration algorithm for the different FEL settings are displayed in Fig. 5. The Gaussian-like distributions of the pulse durations for each setting match the expectations and both probe settings yield consistent results, proving the robustness and versatility of the algorithm. The retrieved temporal profiles of x-ray FEL pulses discloses the expected variation of the SASE intensity substructure within the pulse, providing fundamental and urgently needed information for time-resolved experiments on the femtosecond timescale.

As a demonstration of the huge variety of the pulse shapes and the substructures of SASE FEL pulses revealed within the data analysis, a selection of distinct shots with different pulse durations from $\mathrm{Ne} 1 s$ and Auger data sets is presented in Fig. 6. As one can see from the irregular pulse shape structures, the specification of an rms pulse duration makes much more sense than a full width at half maximum definition, usually applied to ultrashort Gaussian-like pulses.

\section{B. Arrival time determination}

The $\mathrm{x}$-ray-optical synchronization at FEL facilities is limited by an inherent arrival time jitter on the order of $50 \mathrm{fs}$ (FWHM) or larger, deteriorating the time resolution of $\mathrm{x}$ ray-pump-optical-probe experiments [14]. Within the frame of the angular streaking experiments, the x-ray pulses are overlapped with the trailing ramp of the streaking pulse envelope. Therefore, in addition to the pulse structure retrieval, the presented algorithm is also capable of simultaneously measuring the inherent single-shot arrival time jitter of the $\mathrm{x}$-ray pulses with femtosecond resolution. Provided that the envelope of the MIR streaking pulse is initially characterized, this is done by converting the ellipticity-corrected maximum energy shift of all detectors, the streaking kick $a_{\mathrm{ell}}$, into arrival time information for each shot as displayed in Fig. 7.

As mentioned earlier, the streaking kick $a_{\mathrm{ell}}$ is a shotdependent variable due to the inherent arrival time jitter of the $\mathrm{x}$-ray pulses with respect to the MIR field envelope. The latter is Gaussian shaped and has a FWHM duration of $\tau_{\mathrm{MIR}}=$ $480 \mathrm{fs}$. Therefore, the amplitude of the streaking field can be mapped to a Gaussian distribution of the streaking shift, which is described by

$$
a_{\mathrm{ell}}=E_{\max } \exp \left(-\frac{t_{\text {arrival }}{ }^{2}}{2\left(\frac{\tau_{\mathrm{MIR}}}{2.35}\right)^{2}}\right),
$$

where $E_{\max }$ is the maximum possible streaking shift corresponding to the maximum of the MIR amplitude. For even more accuracy, $a_{\text {ell }}$ and $E_{\max }$ are corrected for the shot-toshot power fluctuations of the MIR laser, which were logged 

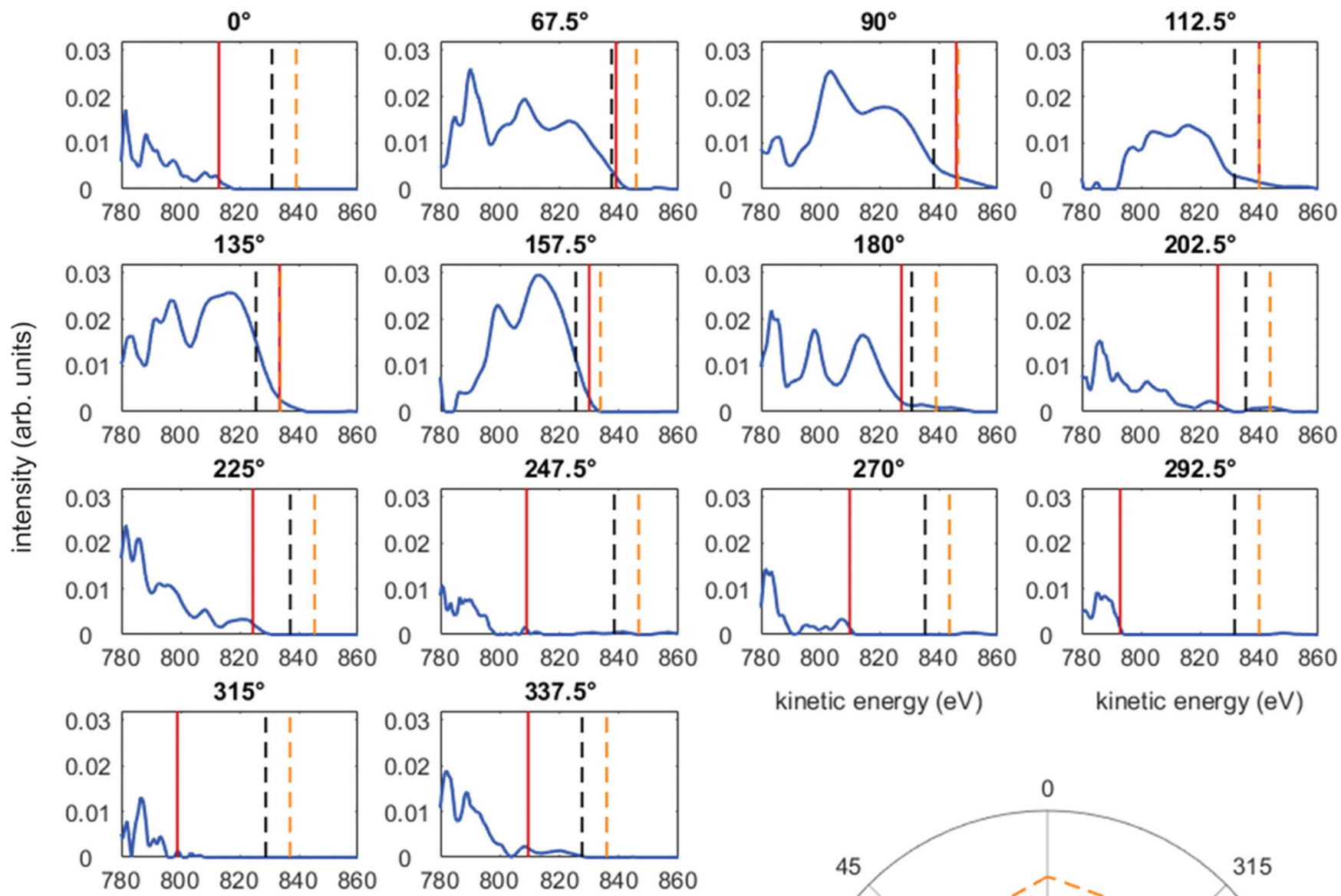

kinetic energy (eV)

kinetic energy (eV)
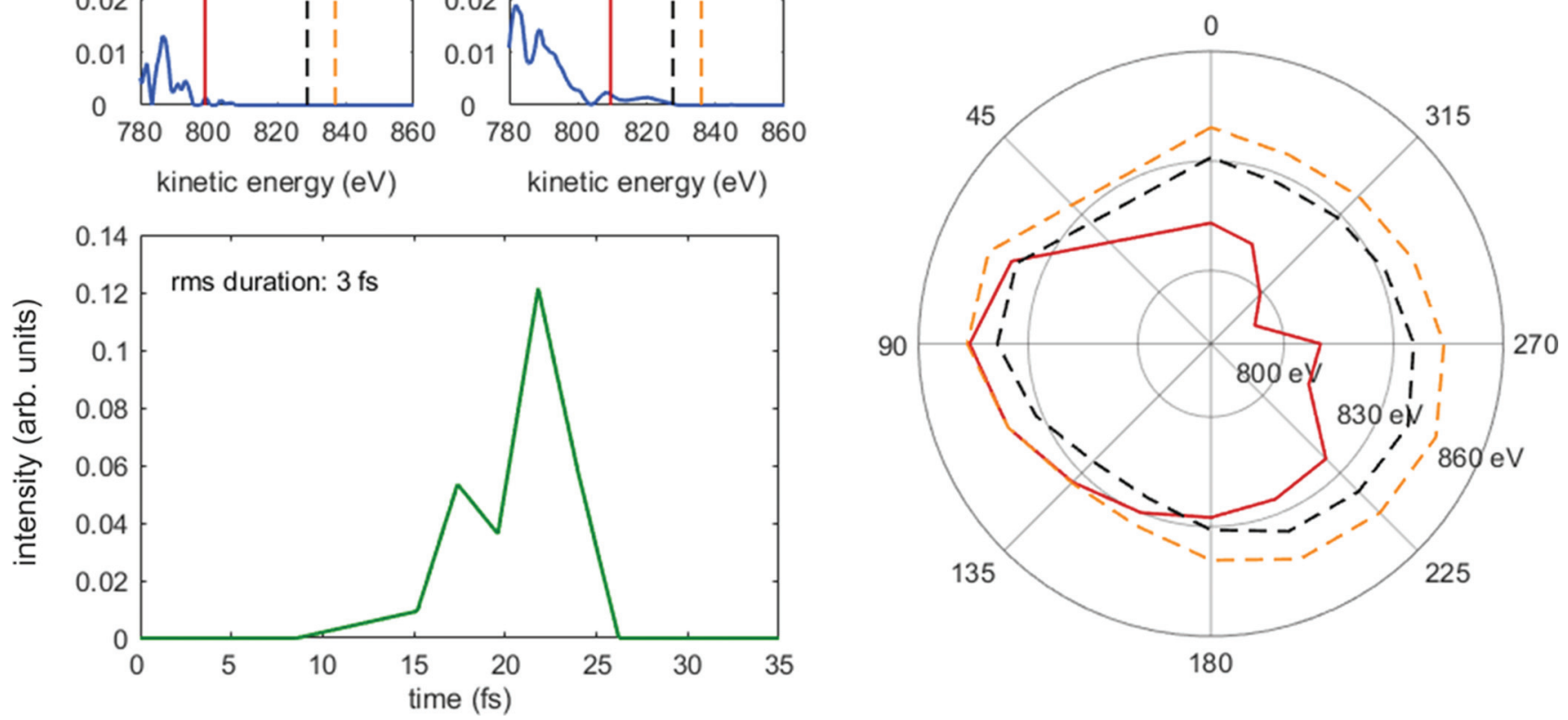

FIG. 3. Compact overview of the integration x-ray pulse retrieval algorithm. Demonstration of the FEL pulse retrieval working principle using one exemplary shot from an Auger data set. The top panels show the streaked spectra (blue lines) of the 14 individual detectors that have been recorded simultaneously for this shot (two detectors at $22.5^{\circ}$ and $45^{\circ}$ had to be excluded from the analysis for this data set due to an electronic shortcut in their retardation voltage wiring). The calculated, angle-dependent energy values $E_{\text {cut }}(\varphi)$ (vertical red full line), $E_{\text {up }}(\varphi)$ (vertical yellow dashed line), and $E_{\text {low }}(\varphi)$ (vertical black dashed line) are marked. These values are displayed additionally in the panel at the bottom, right position in form of a polar plot. The maximum streaking shift at a detection angle of $135^{\circ}$ is clearly visible. The resulting x-ray intensity $I_{\mathrm{x}-\text { ray }}(\varphi)$ is then obtained by integrating the spectral intensity $S(E, \varphi)$ in the range between $E_{\text {low }}(\varphi)$ and $E_{\text {cut }}(\varphi)$, as long as $E_{\text {cut }}(\varphi)>E_{\text {low }}(\varphi)$, whereas it is set to zero in the opposite case. By employing the angular streaking principle, the angle axis is mapped to a temporal axis. The resulting FEL pulse structure $I_{\mathrm{x}-\text { ray }}(t)$ is shown in the bottom, left panel. This shot is made up from two main features and its rms duration is determined to $3 \mathrm{fs}$.

during the experiment. $E_{\max }$ is determined by evaluating an overlap scan, where the temporal delay of the MIR and $\mathrm{X}$-ray pulses has deliberately been varied. For the optimum time overlap, the maximum streaking shift is reached at $E_{\max }=58 \mathrm{eV}$. As the integration retrieval algorithm determines the streaking kick on a single shot basis, the 

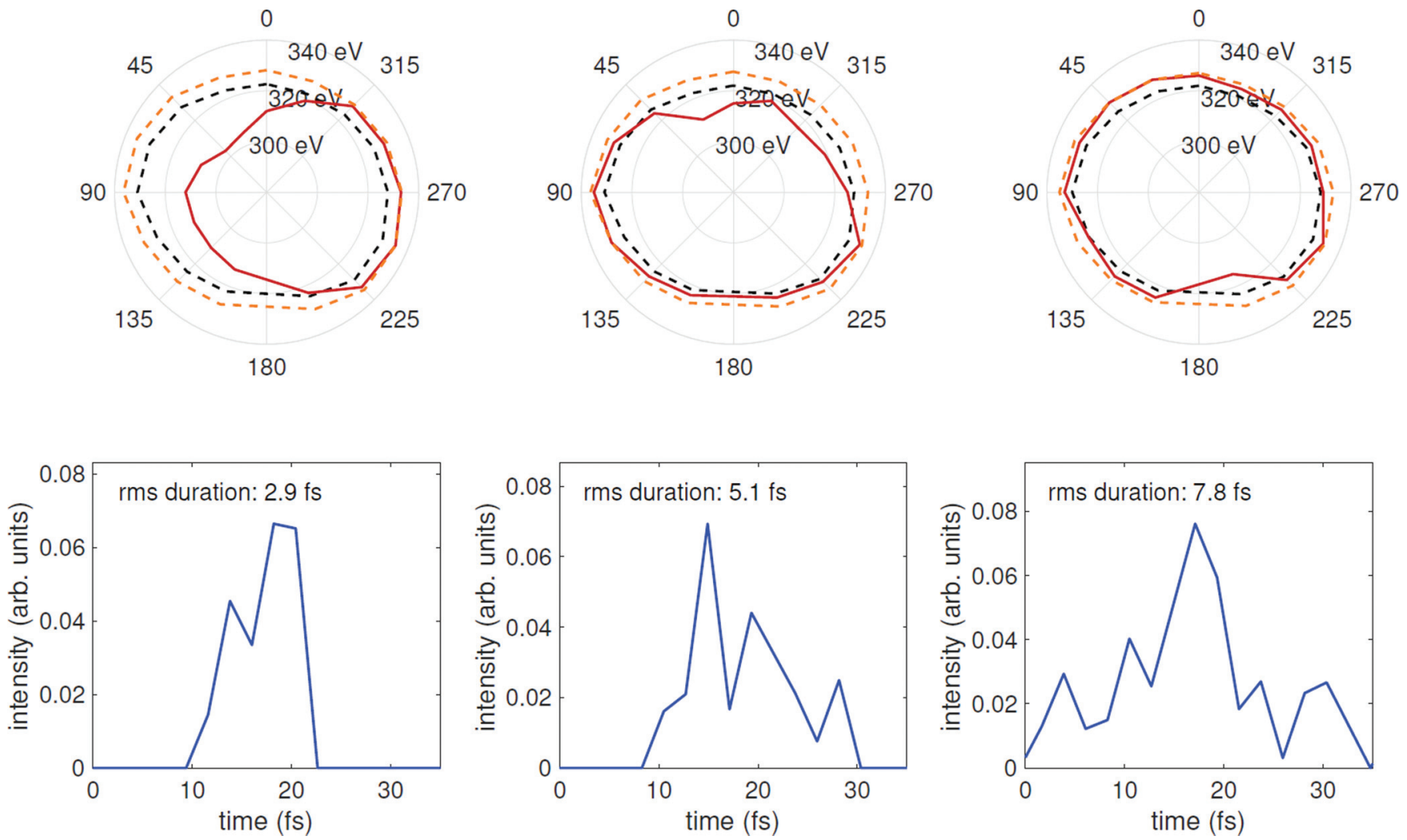

FIG. 4. Retrieval of the pulse substructure and duration for three different pulse duration settings. In the upper panels, the cutoff energy $E_{\text {cut }}(\phi)$ (red full line), as well as the maximum integration window delimited by the lower integration limit $E_{\text {low }}(\phi)$ (black dashed line) and the maximal upper border $E_{\text {up }}(\phi)$ (yellow dashed line), which are deduced from the close-to-circular polarization and the strength of the MIR streaking field, are shown in a polar plot. The spectral intensity $S(E, \varphi)$ between $E_{\text {low }}(\varphi)$ and $E_{\text {cut }}(\varphi)$ is integrated, as it is proportional to the $\mathrm{x}$-ray pulse profile. The corresponding intensity profiles of the x-ray pulses are derived by converting the angular axis to a time axis and are shown in the lower panels. The expected rms pulse durations for the three cases were set to (from left to right) $3.5,6.5$, and 10.5 fs, respectively.
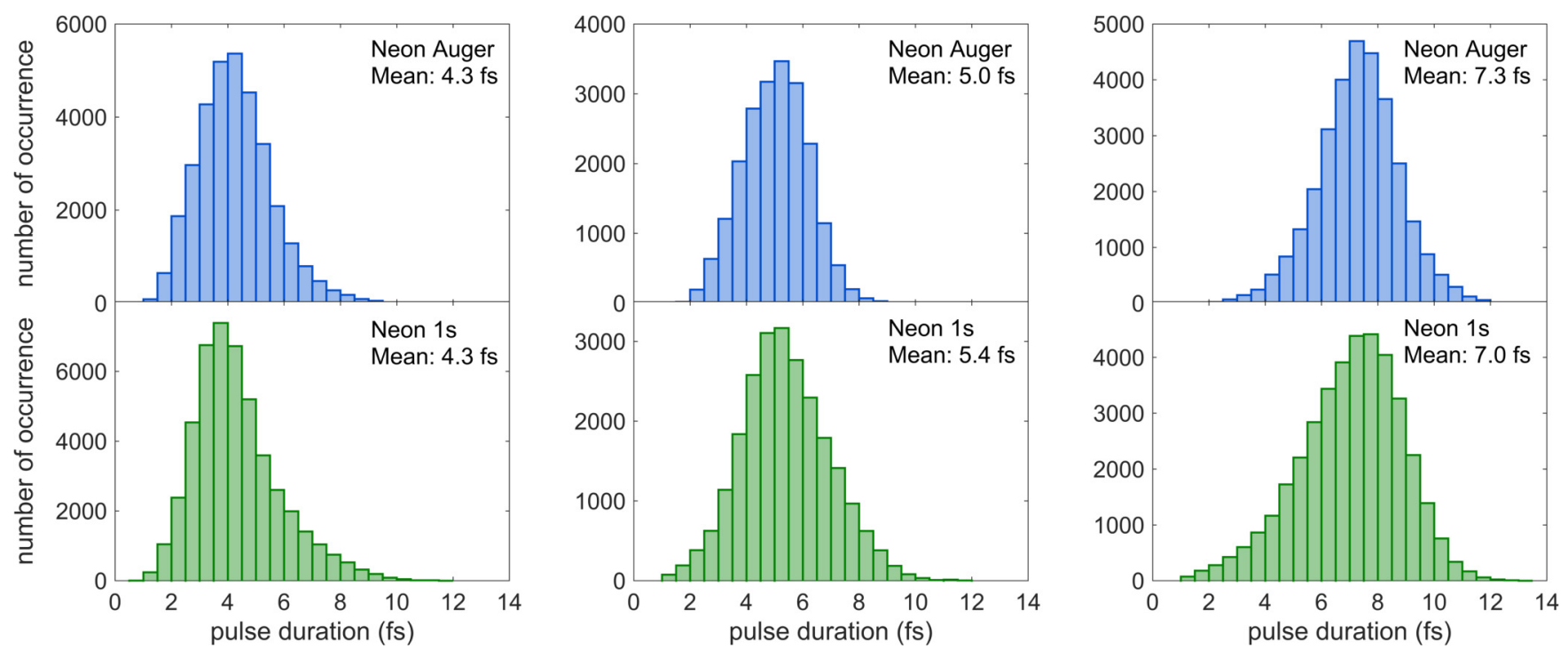

FIG. 5. Histograms of the determined pulse durations. Histograms of the pulse durations and the related mean values derived by the pulse retrieval algorithm for different FEL and detection settings. The upper panels show the results from neon Auger data sets with LCLS pulse duration estimates (rms) of 3.5 (left), 6.5 (middle), and 10.5 fs (right). The lower panels show the corresponding histograms for Ne $1 s$ data sets with the same FEL settings as above. 

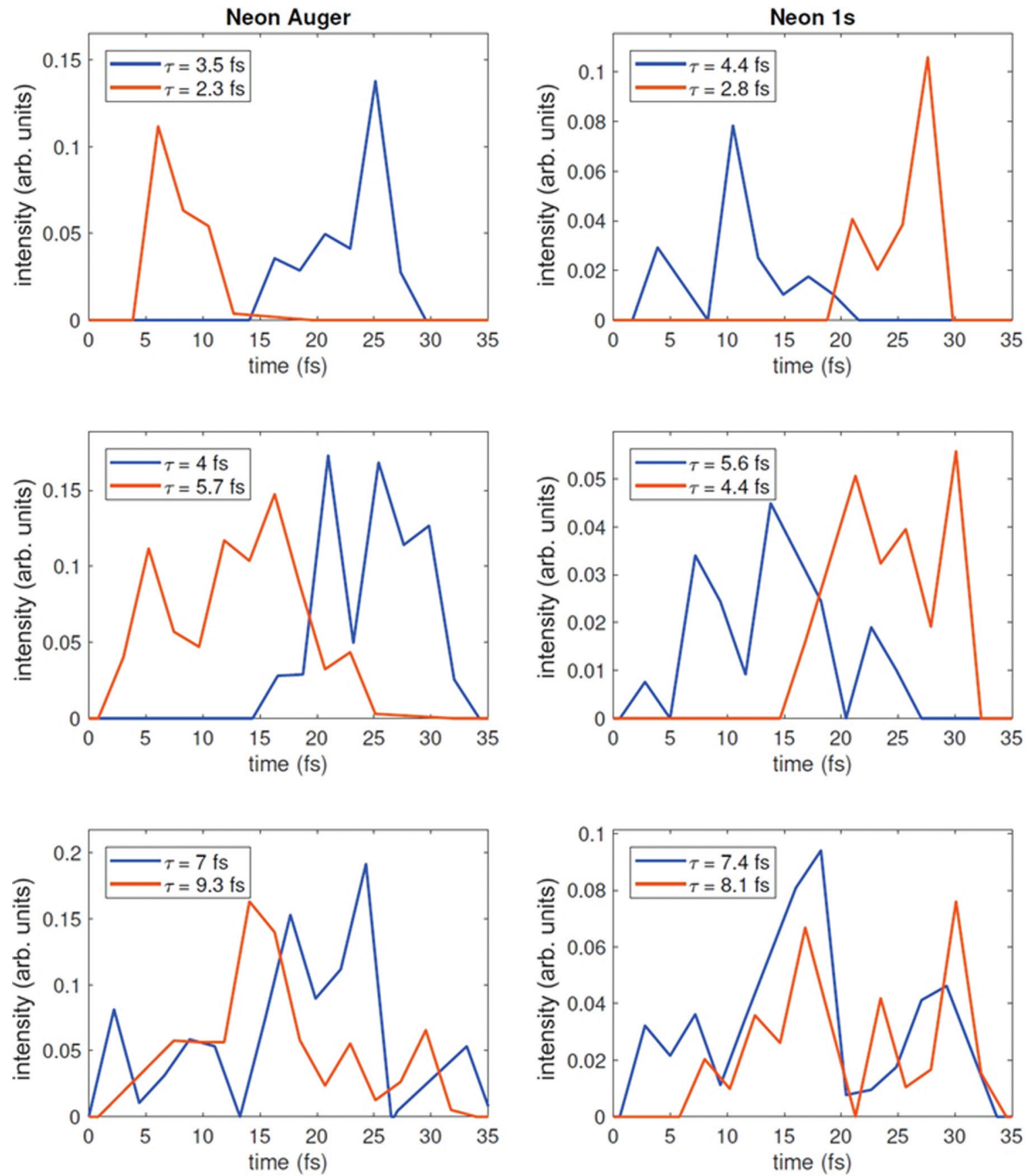

FIG. 6. Variety of the pulse shape and substructure of SASE FEL pulses due to their stochastic nature. A selection of different reconstructed temporal pulse profiles and respective rms pulse durations from Auger data sets (left row) and Ne $1 s$ data sets (right row) is shown. The nominal rms pulse duration settings provided by LCLS for the data set presented in three rows are from top to bottom: 3.5, 6.5, and 10.5 fs. For each setting, two different shots are plotted together in one panel.

arrival time can thus be calculated by

$$
t_{\text {arrival }}=\sqrt{-2 \ln \left(\frac{a_{\mathrm{ell}}}{E_{\mathrm{max}}}\right)\left(\frac{\tau_{\mathrm{MIR}}}{2.35}\right)^{2}} \text {. }
$$

The average jitter is then determined by the FWHM of the

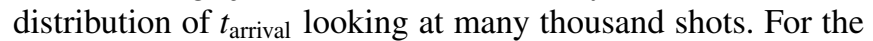
Auger runs evaluated, one obtains an average arrival time jitter of $59 \mathrm{fs}$ FWHM, with an rms resolution of $2.2 \mathrm{fs}$.

\section{Femtosecond online timing unit}

For the single-shot evaluation, we achieve a runtime of approximately $0.5 \mu \mathrm{s}(\triangleq 2 \mathrm{MHz})$ on a quad-core workstation, which can be further reduced by using larger-scale computer clusters. Thus, the angular streaking spectrometer in combination with our fast algorithm can be used as an online noninvasive timing unit with few-femtosecond resolution. In corroboration of the fidelity and the capabilities of our fast integration pulse characterization routine, we applied 

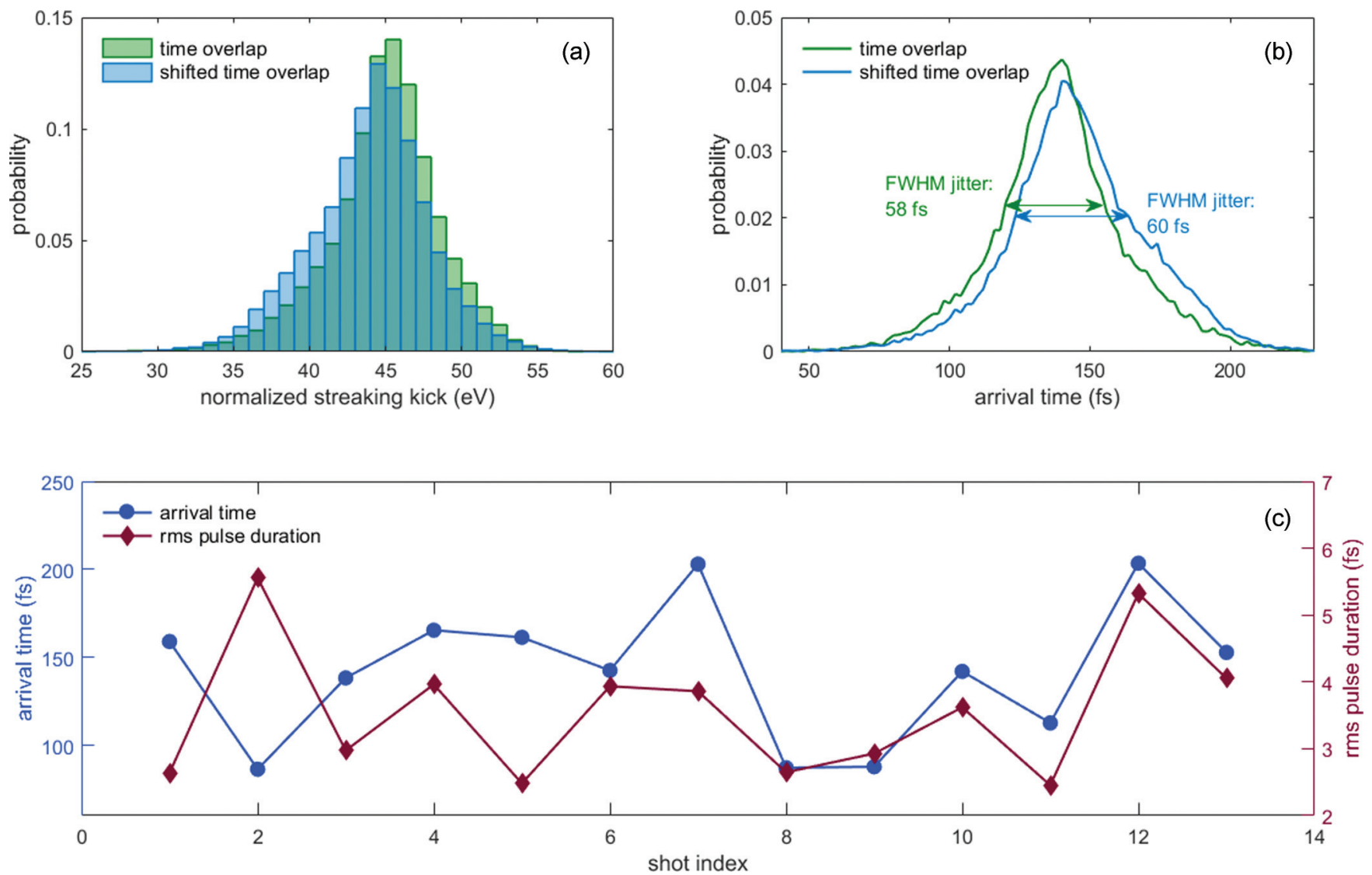

FIG. 7. Determination of the relative x-ray-optical arrival time and the arrival time jitter. (a) The distribution of the retrieved streaking kick $a_{\text {ell }}$, corrected for the MIR power fluctuations, is shown for many thousand shots at two different time overlap positions. The shift to lower energies can be clearly observed while moving out of the optimum temporal overlap. The streaking kick can be converted to the arrival time (b) of the x-ray pulses after a calibration with an MIR-X-ray overlap scan, from which the maximum streaking amplitude and the pulse duration of the MIR laser can be extracted. Here the relative arrival time is given with respect to the maximum of the MIR envelope. The width of these distributions (b) is a measure for the arrival time jitter. (c) The results for the pulse duration (in red) and the arrival time (in blue) are illustrated for a series of consecutive single shots.

both our algorithm and the recently published, iterative and CPU-intensive algorithm achieving a precise attosecond timefrequency characterization of X-ray pulses [30] to an identical angular streaking data set containing a random selection of shots. The benchmark against the iterative algorithm (see Appendix H) further proves the high confidence level of the integration algorithm at orders of magnitude lower computation time and, hence, its applicability as an online tool for pulse characterization.

\section{X-ray double pulses}

In addition to the measurements of isolated x-ray pulses, we also performed angular streaking measurements on $\mathrm{x}$-ray double-pulse trains, generated by the insertion of a doubleslotted foil [49] into the electron bunch of the accelerator before the undulator. X-ray double pulses have already been characterized using terahertz streaking with a lower limit for the resolvable pulse delay of $38 \mathrm{fs}[26,50]$. For our measurements, the average pulse duration was set to $3.5 \mathrm{fs}$ (rms) for both pulses with a temporal delay of about 20 fs between the individual pulses. Additionally to the individual pulse durations and shapes, the separation between the two pulses shall be hereby determined. The integration pulse characterization algorithm is adapted concerning the calculation of the rms widths for the pulse durations of two individual pulses and extended by an additional part for the determination of the separation between the two pulses. A detailed description of the procedure is given in Appendix F.

The results of the extended retrieval algorithm for doublepulse trains applied to many thousand shots are collectively presented for the $\mathrm{Ne} 1 s$ and $\mathrm{Ne}$ Auger electron detection schemes in Fig. 8, showing a shorter mean pulse separation than ever measured before. The substructure of the two individual pulses and their separation are clearly resolved on a single-shot basis [Figs. 8(a) and 8(b)]. The retrieved relative double-pulse separation fits very well to the estimated average value provided by LCLS, revealing a mean pulse separation of 23 fs [Fig. 8(c)]. Smaller pulse separations can straightforwardly be determined by the presented technique through a variation of the streaking laser rotation period.

\section{DISCUSSION}

Within the scope of this work, we present an approach for online FEL pulse characterization with few-femtosecond 

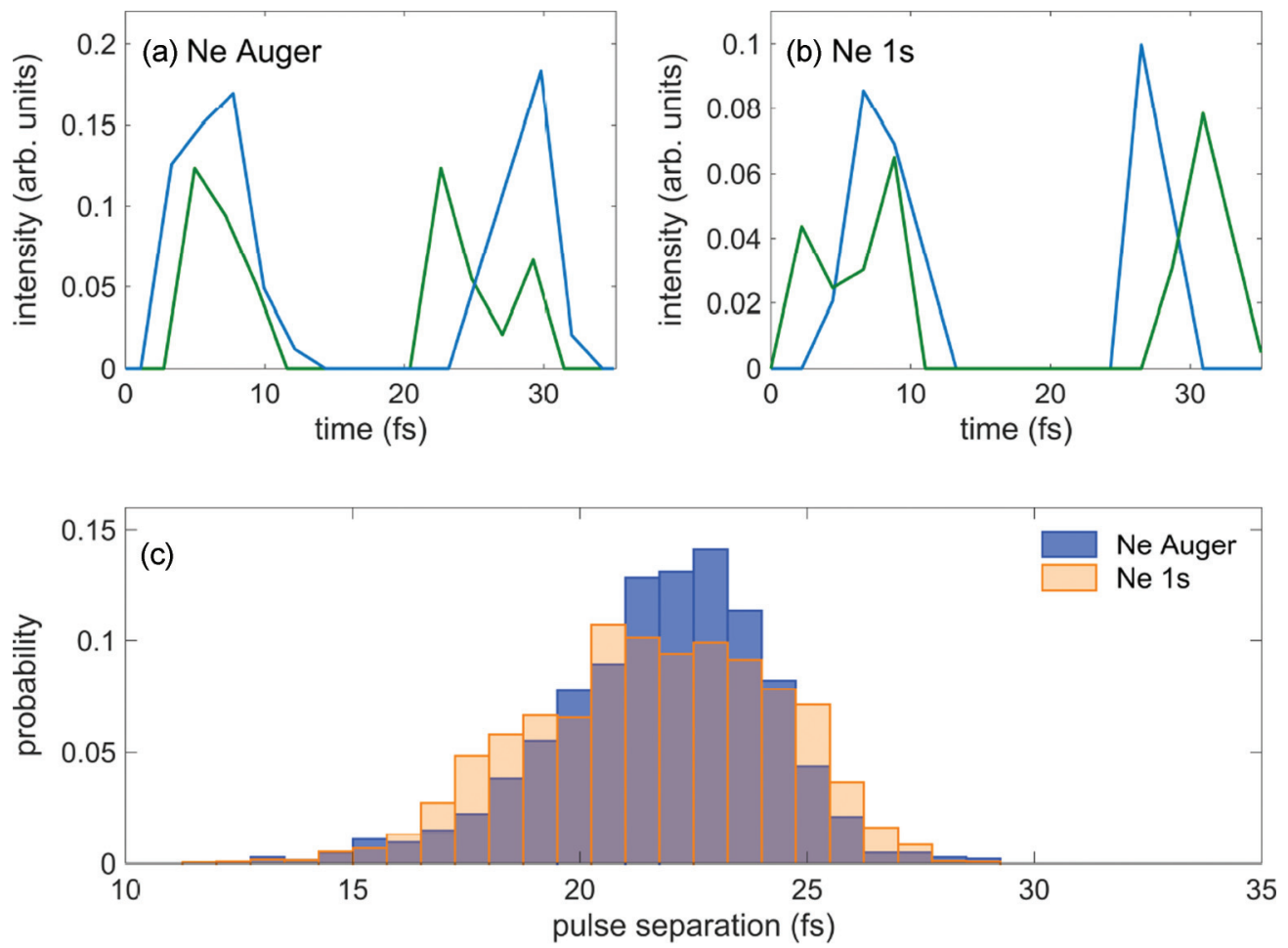

FIG. 8. Temporal characterization of x-ray FEL double-pulse trains. Results of the pulse retrieval algorithm for Ne Auger and Ne $1 s$ double-pulse data sets with an LCLS-estimated pulse separation of $20 \mathrm{fs}$. (a) and (b) The retrieved signals for two exemplary, well-separated double pulses are given in blue and green. (c) The distributions of the relative separations between the two individual peaks of the double pulses for a few thousand shots and both data sets, respectively.

resolution. It provides the possibility to retrieve the temporal intensity profile, including variations in the stochastically changing substructure, and the relative arrival time of single SASE x-ray FEL pulses noninvasively on a single-shot basis. Either Auger electrons or photoelectrons, producing remarkably consistent results, can be used to measure the profile of single FEL pulses with a resolution of about $3.5 \mathrm{fs}$, without substantial x-ray beam intensity losses and independent from simultaneously ongoing experiments. The kinetic energy of the Auger electrons is largely independent of the ionizing photon energy when working well above the respective binding energy threshold, and their emission is approximately isotropic, mostly unsusceptible to the x-ray polarization. In essence this allows for a standardized yet very flexible timing unit regardless of the actual XFEL parameters, working over a broad range of photon energies, polarization states, and pulse durations.

In comparison to the recently presented powerful method for attosecond time-energy reconstruction of FEL pulses, the presented technique requires orders of magnitude less computation time. With its actual repetition rate of $2 \mathrm{MHz}^{1}$, the applicability of this approach as a fast, yet reliable, online tool for pulse characterization is demonstrated. Furthermore, it is feasible to use the presented technique as a pulse vetoing tool allowing us to restrict the data acquisition to measurements with x-ray pulses of specific, desired characteristics. Such

\footnotetext{
${ }^{1}$ Executed on a $3.6 \mathrm{GHz}$ quad-core workstation, see Appendix G.
}

a smart veto logic is urgently required for the implementation of real-time pulse tagging not only at every modern XFEL facility currently existing worldwide, but even at the next-generation XFEL facilities such as the European XFEL or LCLS-II [31,32], which promise to operate at higher repetition rates up to $1 \mathrm{MHz}$. Thus, by only recording data when a few-femtosecond $\mathrm{x}$-ray pulse with desired pulse shape and duration is detected, the shot sorting simultaneously alleviates the need for full-rate detector and data systems. In this respect, this technique is the only one currently available.

Usage of a streaking field with smaller rotation period and/or enhancement of angular resolution can advance this technique's resolution to the attosecond timescale. With exact profiling of SASE pulses available during ongoing measurements, the significance and precision of few-femtosecond $\mathrm{x}$ ray imaging experiments can be substantially improved by incorporating the $\mathrm{x}$-ray substructure intensity distribution into the analysis process. In addition, the characterization of x-ray FEL double-pulse trains clearly reveals the substructure of the two individual pulses on a single-shot basis. Therefore, single-shot knowledge of the separation and the single-peak pulse duration of $\mathrm{x}$-ray double pulses paves the way for highrepetition rate studies of correlation dynamics on the femtosecond time scale by $\mathrm{x}$-ray-pump-X-ray-probe experiments with an adjustable delay.

\section{ACKNOWLEDGMENTS}

This research was carried out at the Linac Coherent Light Source (LCLS) at the SLAC National Accelerator 
Laboratory. Use of the Linac Coherent Light Source (LCLS), SLAC National Accelerator Laboratory, is supported by the U.S. Department of Energy, Office of Science, Office of Basic Energy Sciences under Contract No. DE-AC02-76SF00515. W.H. acknowledges financial support from a Marie Curie International Outgoing Fellowship. W.H., R.H., M.S.W., and R.K. acknowledge financial support by the $\mathrm{BaCaTeC}$ program, the German Cluster of Excellence MAP and the European I3-Initiative "LASERLAB-Europe IV". R.K. acknowledges an ERC Consolidator Grant "AEDMOS". J.K., A.A.L, T.M., S.M., R.N.C., and J.R. acknowledge DOE support under contract DE-AC02-76SF00515. N.M.K. acknowledges financial support from the Theory group in cooperation with the SQS work package of European XFEL. A.K.K. acknowledges financial support from the project FIS2016-76617-P of MINECO. T.F. acknowledges financial support from the National Center of Competence in Research, Molecular Ultrafast Science and Technology. M.I. acknowledges funding of the Volkswagen foundation within a Peter Paul EwaldFellowship.

R.H. and M.S.W. contributed equally to this work. N.H., M.I., A.O.L., R.N.C., and W.H. conceived and coordinated the experiment. N.H., R.H., M.S.W., J.L., A.A.M., S.P.M., J.R., R.N.C., and W.H. built the vacuum system and the optical setup, and supervised them during the measurement. M.I., J.B., A.O.L., J.G., M.P., and J.V. were responsible for constructing, installing, and operating the photoelectron detector. J.K., A.A.L., and T.M. carried out accelerator control and XFEL characterization. R.H., M.S.W., V.S., G.H., M.O., and W.H. performed data analysis. R.H., M.S.W., T.F., R.K., G.H., M.I., N.H., and W.H. interpreted data and oversaw manuscript production. R.H., M.S.W., and W.H. wrote the paper with extensive contributions from all authors.

\section{APPENDIX A: ANGULAR STREAKING}

Using ultrashort FEL pulses neon atoms are ionized and thus electrons are generated in the target region. The rotating electric field of the close-to-circularly polarized infrared streaking laser is used to deflect the emitted electrons in the angular spatial direction. Within a $360^{\circ}$ rotation of the field its amplitude is nearly constant due to the shallow slope of the field envelope for the long IR streaking pulse. The moment of ionization is translated into the angle-dependent kinetic energy shift of the streaked electrons in the IR polarization plane. The rotating electric field can be seen as the hand of clock and the time for one revolution is determined by the carrier period of the infrared laser. In our case, this "attoclock" is set to a $35.3 \mathrm{fs}$ rotation period corresponding to the streaking laser wavelength of $10.6 \mu \mathrm{m}$, in order to be longer than the expected pulse durations of the FEL pulses and to avoid ambiguity from multiple revolutions of the clock within one FEL pulse. The kinetic energy of the photoionized electrons $W\left(W_{0}, \varphi, t_{i}\right)$ is a function of the initial electron energy $W_{0}$ offset by the streaking kick $a$ in the direction of the vector potential at the instant of ionization $t_{i}$ and the observation angle $\varphi$ :

$$
W\left(W_{0}, \varphi, t_{i}\right) \propto W_{0}+a \cos \left(\varphi-2 \pi \frac{c t_{i}}{\lambda}\right) .
$$

Chirp and spectral bandwidth of the $\mathrm{x}$ rays determine the primary electron energy distribution. The photoelectron counts are directly proportional to the actual x-ray intensity.

\section{APPENDIX B: FEL MACHINE SETTINGS AND PARAMETERS}

The FEL is operated at $120 \mathrm{~Hz}$ with an average electron beam energy of $5087 \mathrm{MeV}$ and a peak current of $\sim 1500 \mathrm{~A}$ in a low charge mode with a charge of roughly $120 \mathrm{pC}$. The length of the electron bunch is $85 \mathrm{fs}$, but due to the insertion of the emittance spoiler (slotted foil) the effective lasing part of the bunch is distinctly shorter. For the angular streaking of $\mathrm{Ne} 1 s$ core and $K-L L$ Auger electrons the nominal root-mean-squared (rms) pulse duration of the FEL is set to $3.5,6.5$, and 10.5 fs by tuning the slotted foil position. These specifications on the mean pulse durations have been provided by LCLS and are indirectly determined using a transverse deflection cavity (XTCAV). The emitted x-ray pulses have an average pulse energy of 60,140 , and $180 \mu \mathrm{J}$, respectively, corresponding to the three above mentioned positions of the slotted foil, at a photon energy of $1180 \mathrm{eV}$. To achieve a double pulse mode with a pulse duration of roughly $3.5 \mathrm{fs}$ (rms) and an average, estimated pulse separation of $20 \mathrm{fs,}$ the double-slotted foil was used. For all measurements the IR laser pulse energy is measured on a single-shot basis, on average it is adjusted to $38 \mu \mathrm{J}$. For the angular streaking data sets of $\mathrm{Ne} 1 s$ core electrons (single pulse and double pulse data) the maximum retardation voltage of the time-of-flight spectrometers is set to $270 \mathrm{~V}$, and for resolving the neon Auger electrons the maximum voltage is set to $770 \mathrm{~V}$. For more details see the description of the TOF spectrometer in Appendix D. Similarly, for the energy calibration runs of the $\mathrm{Ne} 1 s$ data the FEL is operated in the low charge mode with a charge of about $130 \mathrm{pC}$, an average electron beam energy of $5087 \mathrm{MeV}$, and a peak current of $\sim 1460 \mathrm{~A}$. The estimated photon energy is tuned to four calibration points by a Vernier scan to $1166,1180,1193$, and $1214 \mathrm{eV}$.

\section{APPENDIX C: OPTICAL LASER SETUP}

The optical (IR) laser setup comprises an ultrafast Ti:sapphire amplifier providing pulses at $800 \mathrm{~nm}$ with $30 \mathrm{~mJ}$ pulse energy and $100 \mathrm{fs}$ pulse duration (FWHM) at $120 \mathrm{~Hz}$ repetition rate and a commercial OPA (optical parametric amplifier) system. In the latter, $10.6 \mu \mathrm{m}$ output pulses with a pulse duration of $340 \mathrm{fs}$ (FWHM) and $38 \mu \mathrm{J}$ average pulse energy on target are generated out of the $800 \mathrm{~nm}$ input via difference frequency generation (DFG) of signal and idler. The linear polarization of that output pulses is converted to a close-to-circular polarization with an ellipticity of $0.73 \pm$ 0.2 by means of a reflective phase retarder (known from $\mathrm{CO}_{2}$ lasers). The carrier-envelope phase of the IR laser pulses was not stabilized. Regarding the vertical detector orientation, the major axis of the ellipse is tilted by $22.5^{\circ} \pm 5^{\circ}$. Using a $\mathrm{ZnSe}$ lens (focal length of $150 \mathrm{~mm}$ ) with a $2-\mathrm{mm}$ central aperture the mid-IR beam is focused in the interaction region to a spot size $\left(1 / \mathrm{e}^{2}\right.$ diameter $)$ of $500 \mu \mathrm{m}$. 


\section{APPENDIX D: TIME-OF-FLIGHT SPECTROMETER}

The spectrometer array is assembled from 16 independently working time-of-flight detectors installed and evenly distributed in steps of $22.5^{\circ}$ in the plane perpendicular to the beam propagation axis. The FEL pulses are focused in a dilute neon gas target (interaction region) centered in the acceptance volume of the spectrometer array. The travel range for the electrons is quite short and only amounts to about $14 \mathrm{~cm}$. In order to cover the whole $2 \pi$ angular detection region by simultaneously observing photoemission spectra at 16 equally spaced angles over a full circle, the acceptance volumes of the single flight tubes are overlapping with each other and the X-ray-IR beam. Resulting from the size of the circular entrance aperture and depending on the adjusted deceleration voltage (ranging from 270 to $770 \mathrm{~V}$ in the present experiment) the angular acceptance of each detector has an upper limit of $3.8 \times 10^{-2} \mathrm{sr}$, in accordance with a full aperture angle of $12.6^{\circ}$. Consequently, the detected signal corresponds to an integral over that aperture. In the detectors, a stack of three multichannel plate (MCP) amplifiers with a capacitive outcoupling are assembled. The detector signal is processed via Acqiris DC282 digitizers run at $4 \mathrm{GS} / \mathrm{s}$ in an interleaved mode and at $2 \mathrm{GS} / \mathrm{s}$ in a noninterleaved mode, respectively. A single electron impinging the MCP amplifiers engenders a measurable narrow voltage spike with a FWHM duration shorter than $1 \mathrm{~ns}$. The spectrometer's energy resolution of the method directly depends on the resolvable pulse width and on the obtained time-energy window due to the fact that the timeof-flight of every arriving photoelectron is equivalent to its kinetic energy. The detected streaking signal is mostly spread over roughly $50 \mathrm{~ns}$. Within this time interval several hundreds of hits in the relevant energy range can be recorded without leaving the linear amplification regime, ensuring single-shot spectroscopy. Thus, the number of detected electrons per unit of time-namely $0.25 \mathrm{~ns}$ in bridged Acqiris modeacquired in each channel is translated to the recorded voltage. Under these terms the energy resolution of a single time-offlight detector is experimentally determined to lie between 0.75 to $1 \mathrm{eV}$.

\section{APPENDIX E: NORMALIZATION AND TRANSMISSION CALIBRATION}

Since we compare spectra measured by independent detectors, we have to carefully adjust the relative signal strengths. In case of the normalization of the Auger spectra with respect to each other, an isotropic emission characteristic of the Auger electrons is assumed. The most prominent $K-L L$ Auger peak in unstreaked time-of-flight traces is used as a measure for the normalization level. For each spectrometer the traces of 2.000 shots from a calibration run are averaged and integrated over a 6 ns-broad time interval around the maximum of the Auger peak. All spectra of the single TOF detectors are then normalized to each other by dividing the spectra by the respective integral values as weighting factors. These once determined normalization factors are equally applied to all Auger data sets. A potential energy dependence of the transmission has been checked, it shows to be flat within the spectral range of interest. Thus, the usage of one single

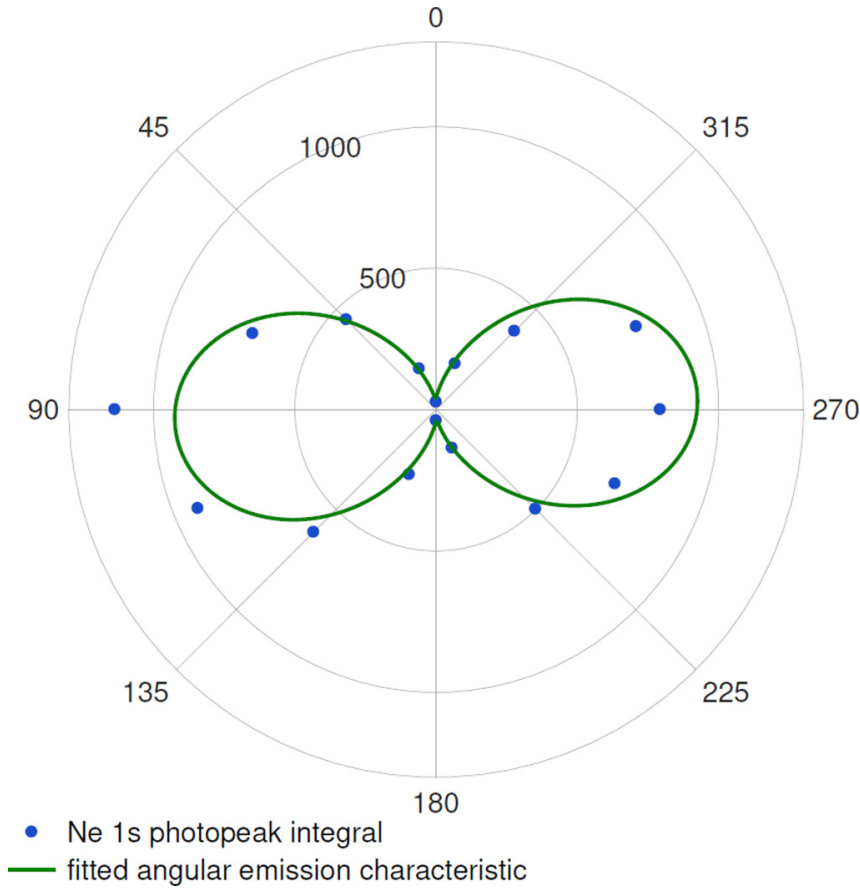

FIG. 9. Correction for the angular emission distribution of $\mathrm{Ne}$ $1 s$ photoelectrons. Ne $1 s$ peak integral values [blue (gray) dots] for the individual e-TOF detectors are fitted [green (gray) line] with the expected dumbbell-shaped angular emission characteristic for ionization with horizontally polarized x-ray pulses from the $\mathrm{Ne} 1 s$ shell. Note the slight tilt of the detector axes with respect to the x-ray polarization plane, leading to a nonzero signal in the $0^{\circ}$ and $180^{\circ}$ TOF detectors.

factor for the whole spectrum for each detector is justified. For the normalization of the photoelectron data sets one uses the $\mathrm{Ne} 1 s$ peak for the integration of the signal to get the normalization weighting factors. The integration window has a width of $4 \mathrm{~ns}$. The angular distribution of the detected $\mathrm{Ne}$ $1 s$ electron counts is dumbbell-shaped with minima at $0^{\circ}$ and $180^{\circ}$ and maxima at $90^{\circ}$ and $270^{\circ}$. This $\beta=2$ emission anisotropy results from the horizontal, linear polarization of the FEL beam and the spherical symmetry of the atomic $1 s$ orbital [51]. The emission characteristic of the $\mathrm{Ne} 1 s$ core electrons is thus reflected by the scaling factors, as can be seen in Fig. 9. This is verified by fitting these normalization factors, calculated via averaged integration over the main photopeak for each individual e-TOF detector, with the differential cross section for the angular emission characteristic

$$
\beta(\theta)=\frac{A}{2}\{1-\cos [2(\theta-B)]\}+C,
$$

where $\theta$ is the detection angle and $A=883.7, B=3.2^{\circ}$, and $C=41.9$ are the fitted parameters for amplitude, an angle offset, and a directional emission shift. Due to good agreement of the fit to the data, the single detectors are normalized to each other by division of the single-shot spectra by the experimentally determined normalization factors in the energy domain. In the same way as for the Auger data, the determined normalization factors are equally applied to all $\mathrm{Ne} 1 s$ data sets. 


\section{APPENDIX F: EXTENDED PROCEDURE FOR THE DOUBLE-PULSE CHARACTERIZATION}

The following procedure is equally applied to both the $\mathrm{Ne} 1 s$ and the Auger data sets. As a first step, a standard peak finding routine using first and second derivatives of the intensity signal is applied. For a clear division into two prominent pulses, it is assumed that the signal between two intensity peaks must drop to at least $5 \%$ of its maximum for at least $2 \mathrm{fs}$, corresponding to the time covered by the angular range of one detector. All FEL shots, where more or less than two prominent peaks are found by the routine, are discarded to select only those shots that would be most suitable for typical pump-probe experiments. Nevertheless, the routine indeed allows shots where one or both pulses consist of more than a single intensity spike, as long as they are clearly separated. The edges of both individual pulses must be determined to reasonably calculate the pulse durations within these bounds for each pulse. We define the outer edges (rising edge of the first pulse and the falling edge of the second pulse) of the double pulses by the value where the peak intensity of the respective pulse has dropped to less than $5 \%$ on each side of the pulse. Instead of the inner edges of the pulses the separation position determined is set to the center of the range between the two pulses, where the intensity stays below $5 \%$ of its maximum for at least $2 \mathrm{fs}$. The separation between the individual pulses is defined as the temporal distance of the centers of mass of both pulses, which is calculated within the defined bounds of the pulses.

\section{APPENDIX G: REQUIRED COMPUTING TIME AND COMPUTATIONAL POWER}

Initially, the source code for the integration algorithm was written in MATLAB and optimized concerning performance. Using the internal MATLAB stopwatch timer for chronometry, the MATLAB routine exhibits a runtime of $58 \mu$ s per shot $(\triangleq 17 \mathrm{kHz}$ repetition rate) on a standard quad-core (3.6 GHz) workstation. The runtime measurement basically comprises solely the single-shot pulse retrieval part of the algorithm. The coefficients for the energy calibration and the polarization ellipse as well as the normalization factors for the detectors have to be statistically determined by averaging thousands of shots in a calibration run a priori. Therefore, these parameters are considered as prerequisites for the pulse shape retrieval and their determination is not included in the runtime measurement. For obtaining a meaningful value for an online, single-shot runtime, these calibration parameters together with the raw time-of-flight data are preloaded in the main memory. Eventually, the runtime of the routine has been even further reduced by a translation from MATLAB to a $\mathrm{C}$ code. This $\mathrm{C}$ version of the retrieval algorithm achieves a runtime of $0.47 \mu$ s per shot, corresponding to a repetition rate of roughly $2.1 \mathrm{MHz}$. As a stopwatch for the execution of the $\mathrm{C}$ code the CPU time has been used. Additionally, for a cross check, the single-shot runtime has been determined by measuring the runtime for a few thousand shots through the real-time clock. Both methods delivered comparable results.

\section{APPENDIX H: PROOF OF CONCEPT-COMPARISON WITH RESULTS FROM AN ITERATIVE RETRIEVAL}

Our here presented fast integration algorithm as well as the recently published time-energy characterization [30] are applied to an identical angular streaking $\mathrm{Ne} 1 s$ data set, containing a random selection of recorded FEL shots, in order to provide the single-shot pulse duration and to reconstruct the temporal pulse substructure. The nominal, averaged pulse duration of the X-ray FEL pulses is set to about $3.5 \mathrm{fs}$ (rms) for all the selected shots. The data set contains only FEL shots that overcome all filter routines mentioned before. Both algorithms are applied independently to raw data of about 46500 FEL shots. Then, the reconstructed pulse intensity profiles of the SASE XFEL pulses, as well as the calculated pulse durations, are comparatively evaluated. For four exemplary x-ray pulses, the reconstructions of the integration pulse characterization algorithm and the established, iterative timeenergy characterization algorithm are comparatively shown in Figs. 10(a)-10(d). For an even better, more reasonable comparability, the high-resolution retrieval of the established time-energy characterization algorithm has been additionally convoluted with a Gaussian response function corresponding to a temporal resolution of $3.5 \mathrm{fs}$. This convolution is applied in order to reflect the temporal resolution of the integration algorithm. Overall, these results verify our time resolution once again. Both algorithms reveal very consistent results on the reconstructed substructure of the individual X-ray pulses composed of SASE spikes of varying number and shape. Also the respectively calculated pulse durations are very similar. The good agreement proves the validity of the described fast integration procedure. For a more detailed evaluation of the similarity of the pulse substructures retrieved by both routines for the same SASE pulse, additional statistical methods are applied. The reconstructed intensity profiles from the integration algorithm have been cross correlated with the convoluted temporal reconstruction of the iterative time-energy retrieval for each shot in order to optimize the overlap of both pulse shapes on the time axis within an interval of $\pm 2.2 \mathrm{fs}$, corresponding to the detector spacing. The Pearson correlation coefficient

$$
\rho(A, B)=\frac{1}{N-1} \sum_{i=1}^{N}\left(\frac{A_{i}-\mu_{A}}{\sigma_{A}}\right)\left(\frac{B_{i}-\mu_{B}}{\sigma_{B}}\right)
$$

of the two reconstructed intensity profiles $A$ (integration algorithm) and $B$ (iterative algorithm) is determined for each shot of the data set. Here $\mu_{A}$ and $\sigma_{A}$ are the mean and standard deviation of $A$ and $\mu_{B}$ and $\sigma_{B}$ are the mean and standard deviation of $B$, respectively. The according distribution is displayed in Fig. 10(f). The median of the correlation coefficient amounts to 0.75 , constituting a strong correlation between the reconstructed pulse shapes. Furthermore, the distributions of the calculated rms pulse durations, delivered from both algorithms, are comparatively displayed for all shots of the data set in Fig. 10(e). Regarding the integration algorithm, the mean of the rms pulse duration distribution amounts to $4.4 \mathrm{fs}$, while the mean value for the iterative time-energy characterization algorithm is calculated to $3.4 \mathrm{fs}$. Both results are close to the nominal, averaged pulse duration of $3.5 \mathrm{fs}$, provided by LCLS. The constraints of the temporal resolution of the integration algorithm are visualized by the shift of the two distributions to each other. 

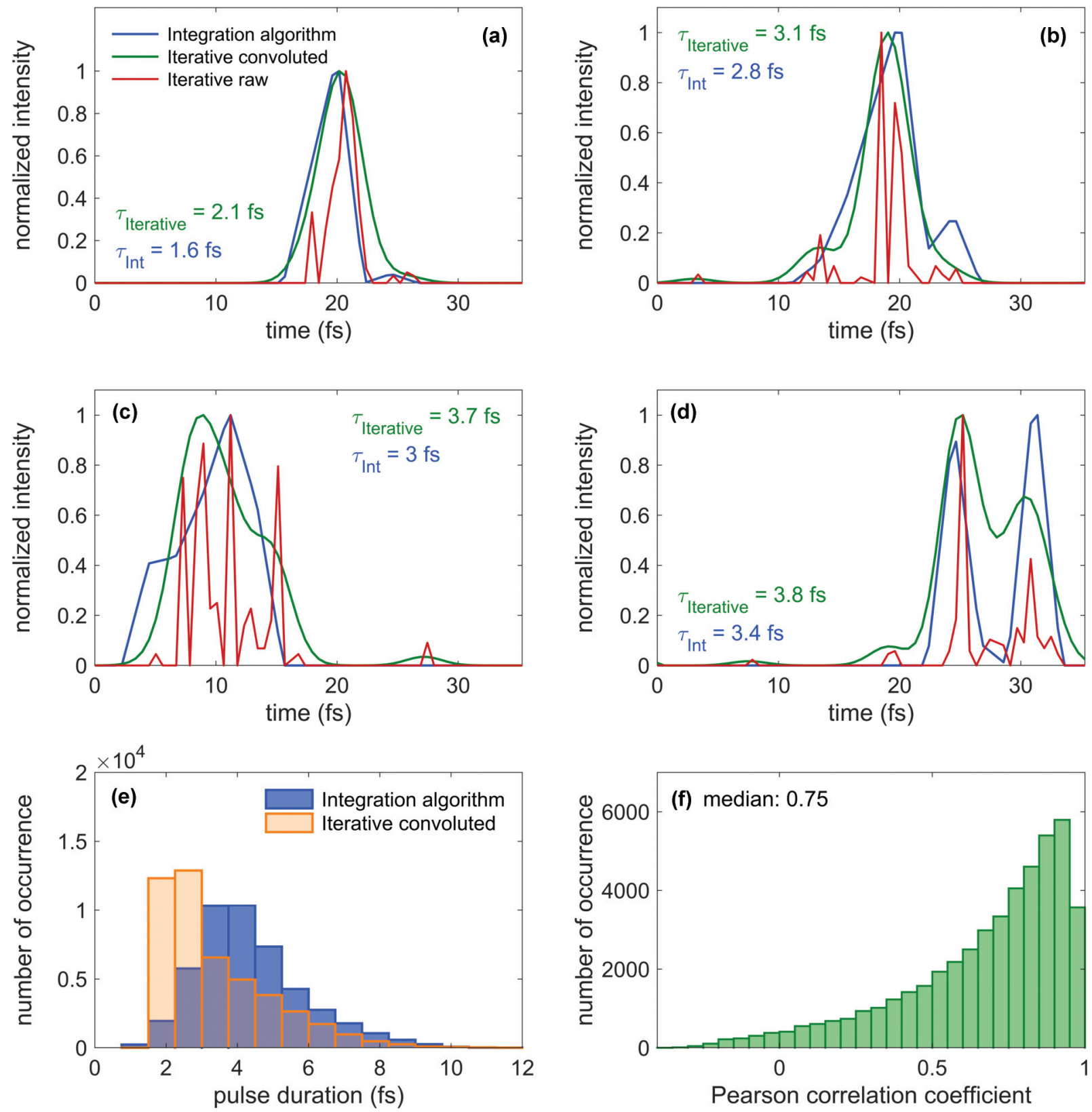

FIG. 10. Evaluation of the integration algorithm by a benchmarking test with the iterative algorithm. A comparison of the reconstructed temporal intensity profiles of exemplary x-ray FEL pulses is presented (a)-(d). The respective reconstruction of both algorithms, as well as the reconstruction of the iterative algorithm convoluted with a Gaussian response function are displayed for four x-ray FEL pulses. The according rms pulse durations determined for both reconstructions are also denoted. (e) The histograms of the distributions of the rms pulse durations determined by both algorithms are plotted. Despite a small shift due to the temporal resolution of the integration algorithm, the distributions show a good agreement. (f) The distribution of the Pearson correlation coefficient is illustrated, together with its median.

[1] P. Emma, R. Akre, J. Arthur, R. Bionta, C. Bostedt, J. Bozek, A. Brachmann, P. Bucksbaum, R. Coffee, F.-J. Decker, Y. Ding, D. Dowell, S. Edstrom, A. Fisher, J. Frisch, S. Gilevich, J. Hastings, G. Hays, P. Hering, Z. Huang, R. Iverson, H. Loos, M. Messerschmidt, A. Miahnahri, S. Moeller, H.-D. Nuhn, G. Pile, D. Ratner, J. Rzepiela, D. Schultz, T. Smith, P. Stefan, H. Tompkins, J. Turner, J. Welch, W. White, J. Wu, G. Yocky, and J. Galayda, First lasing and operation of an ångstrom-wavelength free-electron laser, Nat. Photon. 4, 641 (2010).
[2] T. Ishikawa, H. Aoyagi, T. Asaka, Y. Asano, N. Azumi, T. Bizen, H. Ego, K. Fukami, T. Fukui, Y. Furukawa, S. Goto, H. Hanaki, T. Hara, T. Hasegawa, T. Hatsui, A. Higashiya, T. Hirono, N. Hosoda, M. Ishii, T. Inagaki, Y. Inubushi, T. Itoga, Y. Joti, M. Kago, T. Kameshima, H. Kimura, Y. Kirihara, A. Kiyomichi, T. Kobayashi, C. Kondo, T. Kudo, H. Maesaka, X. M. Maréchal, T. Masuda, S. Matsubara, T. Matsumoto, T. Matsushita, S. Matsui, M. Nagasono, N. Nariyama, H. Ohashi, T. Ohata, T. Ohshima, S. Ono, Y. Otake, C. Saji, T. Sakurai, T. Sato, K. Sawada, T. Seike, K. Shirasawa, T. Sugimoto, 
S. Suzuki, S. Takahashi, H. Takebe, K. Takeshita, K. Tamasaku, H. Tanaka, R. Tanaka, T. Tanaka, T. Togashi, K. Togawa, A. Tokuhisa, H. Tomizawa, K. Tono, S. Wu, M. Yabashi, M. Yamaga, A. Yamashita, K. Yanagida, C. Zhang, T. Shintake, H. Kitamura, and N. Kumagai, A compact x-ray free-electron laser emitting in the sub-ångström region, Nat. Photon. 6, 540 (2012).

[3] E. Allaria, D. Castronovo, P. Cinquegrana, P. Craievich, M. Dal Forno, M. B. Danailov, G. D’Auria, A. Demidovich, G. de Ninno, S. Di Mitri, B. Diviacco, W. M. Fawley, M. Ferianis, E. Ferrari, L. Froehlich, G. Gaio, D. Gauthier, L. Giannessi, R. Ivanov, B. Mahieu, N. Mahne, I. Nikolov, F. Parmigiani, G. Penco, L. Raimondi, C. Scafuri, C. Serpico, P. Sigalotti, S. Spampinati, C. Spezzani, M. Svandrlik, C. Svetina, M. Trovo, M. Veronese, D. Zangrando, and M. Zangrando, Twostage seeded soft-x-ray free-electron laser, Nat. Photon. 7, 913 (2013).

[4] L. Young, E. P. Kanter, B. Krässig, Y. Li, A. M. March, S. T. Pratt, R. Santra, S. H. Southworth, N. Rohringer, L. F. Dimauro, G. Doumy, C. A. Roedig, N. Berrah, L. Fang, M. Hoener, P. H. Bucksbaum, J. P. Cryan, S. Ghimire, J. M. Glownia, D. A. Reis, J. D. Bozek, C. Bostedt, and M. Messerschmidt, Femtosecond electronic response of atoms to ultra-intense x-rays, Nature (London) 466, 56 (2010).

[5] A. Rudenko, L. Inhester, K. Hanasaki, X. Li, S. J. Robatjazi, B. Erk, R. Boll, K. Toyota, Y. Hao, O. Vendrell, C. Bomme, E. Savelyev, B. Rudek, L. Foucar, S. H. Southworth, C. S. Lehmann, B. Kraessig, T. Marchenko, M. Simon, K. Ueda, K. R. Ferguson, M. Bucher, T. Gorkhover, S. Carron, R. Alonso-Mori, J. E. Koglin, J. Correa, G. J. Williams, S. Boutet, L. Young, C. Bostedt, S.-K. Son, R. Santra, and D. Rolles, Femtosecond response of polyatomic molecules to ultra-intense hard x-rays, Nature (London) 546, 129 (2017).

[6] R. Boll, D. Anielski, C. Bostedt, J. D. Bozek, L. Christensen, R. Coffee, S. De, P. Decleva, S. W. Epp, B. Erk, L. Foucar, F. Krasniqi, J. Küpper, A. Rouzée, B. Rudek, A. Rudenko, S. Schorb, H. Stapelfeldt, M. Stener, S. Stern, S. Techert, S. Trippel, M. J. J. Vrakking, J. Ullrich, and D. Rolles, Femtosecond photoelectron diffraction on laser-aligned molecules: Towards time-resolved imaging of molecular structure, Phys. Rev. A 88, 061402 (2013).

[7] M. W. Mara, R. G. Hadt, M. E. Reinhard, T. Kroll, H. Lim, R. W. Hartsock, R. Alonso-Mori, M. Chollet, J. M. Glownia, S. Nelson, D. Sokaras, K. Kunnus, K. O. Hodgson, B. Hedman, U. Bergmann, K. J. Gaffney, and E. I. Solomon, Metalloprotein entatic control of ligand-metal bonds quantified by ultrafast $\mathrm{X}$ ray spectroscopy, Science 356, 1276 (2017).

[8] N. Coquelle, M. Sliwa, J. Woodhouse, G. Schirò, V. Adam, A. Aquila, T. R. M. Barends, S. Boutet, M. Byrdin, S. Carbajo, E. de La Mora, R. B. Doak, M. Feliks, F. Fieschi, L. Foucar, V. Guillon, M. Hilpert, M. S. Hunter, S. Jakobs, J. E. Koglin, G. Kovacsova, T. J. Lane, B. Lévy, M. Liang, K. Nass, J. Ridard, J. S. Robinson, C. M. Roome, C. Ruckebusch, M. Seaberg, M. Thepaut, M. Cammarata, I. Demachy, M. Field, R. L. Shoeman, D. Bourgeois, J.-P. Colletier, I. Schlichting, and M. Weik, Chromophore twisting in the excited state of a photoswitchable fluorescent protein captured by time-resolved serial femtosecond crystallography, Nat. Chem. 10, 31 (2018).

[9] D. Milathianaki, S. Boutet, G. J. Williams, A. Higginbotham, D. Ratner, A. E. Gleason, M. Messerschmidt, M. M. Seibert,
D. C. Swift, P. Hering, J. Robinson, W. E. White, and J. S. Wark, Femtosecond visualization of lattice dynamics in shock-compressed matter, Science 342, 220 (2013).

[10] C. S. Lehmann, A. Picón, C. Bostedt, A. Rudenko, A. Marinelli, D. Moonshiram, T. Osipov, D. Rolles, N. Berrah, C. Bomme, M. Bucher, G. Doumy, B. Erk, K. R. Ferguson, T. Gorkhover, P. J. Ho, E. P. Kanter, B. Krässig, J. Krzywinski, A. A. Lutman, A. M. March, D. Ray, L. Young, S. T. Pratt, and S. H. Southworth, Ultrafast $\mathrm{x}$-ray-induced nuclear dynamics in diatomic molecules using femtosecond x-ray-pump-x-rayprobe spectroscopy, Phys. Rev. A 94, 013426 (2016).

[11] B. Erk, D. Rolles, L. Foucar, B. Rudek, S. W. Epp, M. Cryle, C. Bostedt, S. Schorb, J. Bozek, A. Rouzee, A. Hundertmark, T. Marchenko, M. Simon, F. Filsinger, L. Christensen, S. De, S. Trippel, J. Küpper, H. Stapelfeldt, S. Wada, K. Ueda, M. Swiggers, M. Messerschmidt, C. D. Schröter, R. Moshammer, I. Schlichting, J. Ullrich, and A. Rudenko, Ultrafast Charge Rearrangement and Nuclear Dynamics upon Inner-Shell Multiple Ionization of small Polyatomic Molecules, Phys. Rev. Lett. 110, 053003 (2013).

[12] H. N. Chapman, P. Fromme, A. Barty, T. A. White, R. A. Kirian, A. Aquila, M. S. Hunter, J. Schulz, D. P. DePonte, U. Weierstall, R. B. Doak, F. R. N. C. Maia, A. V. Martin, I. Schlichting, L. Lomb, N. Coppola, R. L. Shoeman, S. W. Epp, R. Hartmann, D. Rolles, A. Rudenko, L. Foucar, N. Kimmel, G. Weidenspointner, P. Holl, M. Liang, M. Barthelmess, C. Caleman, S. Boutet, M. J. Bogan, J. Krzywinski, C. Bostedt, S. Bajt, L. Gumprecht, B. Rudek, B. Erk, C. Schmidt, A. Hömke, C. Reich, D. Pietschner, L. Strüder, G. Hauser, H. Gorke, J. Ullrich, S. Herrmann, G. Schaller, F. Schopper, H. Soltau, K.-U. Kühnel, M. Messerschmidt, J. D. Bozek, S. P. Hau-Riege, M. Frank, C. Y. Hampton, R. G. Sierra, D. Starodub, G. J. Williams, J. Hajdu, N. Timneanu, M. M. Seibert, J. Andreasson, A. Rocker, O. Jönsson, M. Svenda, S. Stern, K. Nass, R. Andritschke, C.-D. Schröter, F. Krasniqi, M. Bott, K. E. Schmidt, X. Wang, I. Grotjohann, J. M. Holton, T. R. M. Barends, R. Neutze, S. Marchesini, R. Fromme, S. Schorb, D. Rupp, M. Adolph, T. Gorkhover, I. Andersson, H. Hirsemann, G. Potdevin, H. Graafsma, B. Nilsson, and J. C. H. Spence, Femtosecond x-ray protein nanocrystallography, Nature (London) 470, 73 (2011).

[13] T. Tschentscher, Investigation of ultrafast processes using x-ray free-electron laser radiation, Chem. Phys. 299, 271 (2004).

[14] S. V. Milton, E. Gluskin, N. D. Arnold, C. Benson, W. Berg, S. G. Biedron, M. Borland, Y. C. Chae, R. J. Dejus, P. K. Den Hartog, B. Deriy, M. Erdmann, Y. I. Eidelman, M. W. Hahne, Z. Huang, K. J. Kim, J. W. Lewellen, Y. Li, A. H. Lumpkin, O. Makarov, E. R. Moog, A. Nassiri, V. Sajaev, R. Soliday, B. J. Tieman, E. M. Trakhtenberg, G. Travish, I. B. Vasserman, N. A. Vinokurov, X. J. Wang, G. Wiemerslage, and B. X. Yang, Exponential gain and saturation of a self-amplified spontaneous emission free-electron laser, Science 292, 2037 (2001).

[15] C. Behrens, F.-J. Decker, Y. Ding, V. A. Dolgashev, J. Frisch, Z. Huang, P. Krejcik, H. Loos, A. Lutman, T. J. Maxwell, J. Turner, J. Wang, M.-H. Wang, J. Welch, and J. Wu, Few-femtosecond time-resolved measurements of x-ray freeelectron lasers, Nat. Commun. 5, 3762 (2014).

[16] J. P. MacArthur, J. Duris, Z. Huang, and A. Marinelli, High power sub-femtosecond x-ray pulse study for the LCLS, 
Proceedings of 8th Int. Particle Accelerator Conf. (IPAC'17): 2848-2850 (2017).

[17] A. A. Lutman, M. W. Guetg, T. J. Maxwell, J. P. MacArthur, Y. Ding, C. Emma, J. Krzywinski, A. Marinelli, and Z. Huang, High-Power Femtosecond Soft X Rays from Fresh-Slice Multistage Free-Electron Lasers, Phys. Rev. Lett. 120, 264801 (2018).

[18] A. A. Lutman, J. P. MacArthur, M. Ilchen, A. O. Lindahl, J. Buck, R. N. Coffee, G. L. Dakovski, L. Dammann, Y. Ding, H. A. Dürr, L. Glaser, J. Grünert, G. Hartmann, N. Hartmann, D. Higley, K. Hirsch, Y. I. Levashov, A. Marinelli, T. Maxwell, A. Mitra, S. Moeller, T. Osipov, F. Peters, M. Planas, I. Shevchuk, W. F. Schlotter, F. Scholz, J. Seltmann, J. Viefhaus, P. Walter, Z. R. Wolf, Z. Huang, and H.-D. Nuhn, Polarization control in an X-ray free-electron laser, Nat. Photon. 10, 468 (2016).

[19] M. Hentschel, R. Kienberger, C. Spielmann, G. A. Reider, N. Milosevic, T. Brabec, P. Corkum, U. Heinzmann, M. Drescher, and F. Krausz, Attosecond metrology, Nature (London) 414, 509 (2001).

[20] M. Drescher, M. Hentschel, R. Kienberger, G. Tempea, C. Spielmann, G. A. Reider, P. B. Corkum, and F. Krausz, X-ray pulses approaching the attosecond frontier, Science 291, 1923 (2001).

[21] M. Schultze, M. Fiess, N. Karpowicz, J. Gagnon, M. Korbman, M. Hofstetter, S. Neppl, A. L. Cavalieri, Y. Komninos, T. Mercouris, C. A. Nicolaides, R. Pazourek, S. Nagele, J. Feist, J. Burgdörfer, A. M. Azzeer, R. Ernstorfer, R. Kienberger, U. Kleineberg, E. Goulielmakis, F. Krausz, and V. S. Yakovlev, Delay in photoemission, Science 328, 1658 (2010).

[22] M. Ossiander, F. Siegrist, V. Shirvanyan, R. Pazourek, A. Sommer, T. Latka, A. Guggenmos, S. Nagele, J. Feist, J. Burgdörfer, R. Kienberger, and M. Schultze, Attosecond correlation dynamics, Nat. Phys. 13, 280 (2016).

[23] A. L. Cavalieri, N. Müller, T. Uphues, V. S. Yakovlev, A. Baltuska, B. Horvath, B. Schmidt, L. Blümel, R. Holzwarth, S. Hendel, M. Drescher, U. Kleineberg, P. M. Echenique, R. Kienberger, F. Krausz, and U. Heinzmann, Attosecond spectroscopy in condensed matter, Nature (London) 449, 1029 (2007).

[24] R. Kienberger, E. Goulielmakis, M. Uiberacker, A. Baltuska, V. Yakovlev, F. Bammer, A. Scrinzi, T. Westerwalbesloh, U. Kleineberg, U. Heinzmann, M. Drescher, and F. Krausz, Atomic transient recorder, Nature (London) 427, 817 (2004).

[25] W. Helml, A. R. Maier, W. Schweinberger, I. Grguraš, P. Radcliffe, G. Doumy, C. Roedig, J. Gagnon, M. Messerschmidt, S. Schorb, C. Bostedt, F. Grüner, L. F. Dimauro, D. Cubaynes, J. D. Bozek, T. Tschentscher, J. T. Costello, M. Meyer, R. Coffee, S. Düsterer, A. L. Cavalieri, and R. Kienberger, Measuring the temporal structure of few-femtosecond free-electron laser $\mathrm{X}$-ray pulses directly in the time domain, Nat. Photon. 8, 950 (2014).

[26] I. Grguraš, A. R. Maier, C. Behrens, T. Mazza, T. J. Kelly, P. Radcliffe, S. Düsterer, A. K. Kazansky, N. M. Kabachnik, T. Tschentscher, J. T. Costello, M. Meyer, M. C. Hoffmann, H. Schlarb, and A. L. Cavalieri, Ultrafast X-ray pulse characterization at free-electron lasers, Nat. Photon. 6, 852 (2012).

[27] S. Düsterer, P. Radcliffe, C. Bostedt, J. Bozek, A. L. Cavalieri, R. Coffee, J. T. Costello, D. Cubaynes, L. F. Dimauro, Y. Ding, G. Doumy, F. Grüner, W. Helml, W. Schweinberger, R. Kienberger, A. R. Maier, M. Messerschmidt, V. Richardson, C. Roedig, T. Tschentscher, and M. Meyer, Femtosecond
X-ray pulse length characterization at the Linac Coherent Light Source free-electron laser, New J. Phys. 13, 93024 (2011).

[28] U. Frühling, M. Wieland, M. Gensch, T. Gebert, B. Schütte, M. Krikunova, R. Kalms, F. Budzyn, O. Grimm, J. Rossbach, E. Plönjes, and M. Drescher, Single-shot terahertz-field-driven x-ray streak camera, Nat. Photon. 3, 523 (2009)

[29] L. Zhao, Z. Wang, C. Lu, R. Wang, C. Hu, P. Wang, J. Qi, T. Jiang, S. Liu, Z. Ma, F. Qi, P. Zhu, Y. Cheng, Z. Shi, Y. Shi, W. Song, X. Zhu, J. Shi, Y. Wang, L. Yan, L. Zhu, D. Xiang, and J. Zhang, Terahertz Streaking of Few-Femtosecond Relativistic Electron Beams, Phys. Rev. X 8, 021061 (2018).

[30] N. Hartmann, G. Hartmann, R. Heider, M. S. Wagner, M. Ilchen, J. Buck, A. O. Lindahl, C. Benko, J. Grünert, J. Krzywinski, J. Liu, A. A. Lutman, A. Marinelli, T. Maxwell, A. A. Miahnahri, S. P. Moeller, M. Planas, J. Robinson, A. K. Kazansky, N. M. Kabachnik, J. Viefhaus, T. Feurer, R. Kienberger, R. N. Coffee, and W. Helml, Attosecond timeenergy structure of $\mathrm{x}$-ray free-electron laser pulses, Nat. Photon. 12, 215 (2018).

[31] M. Altarelli, The European X-ray free-electron laser facility in Hamburg, Nucl. Instrum. Methods B 269, 2845 (2011).

[32] T. O. Raubenheimer, LCLS-II: Status of the CW x-ray FEL upgrade to the SLAC LCLS facility, Proceedings 37th International Free Electron Laser Conference (FEL'15): 618-624 (2015).

[33] A. K. Kazansky, A. V. Bozhevolnov, I. P. Sazhina, and N. M. Kabachnik, Interference effects in angular streaking with a rotating terahertz field, Phys. Rev. A. 93, 1307 (2016).

[34] A. K. Kazansky, I. P. Sazhina, V. L. Nosik, and N. M. Kabachnik, Angular streaking and sideband formation in rotating terahertz and far-infrared fields, J. Phys. B. 50, 105601 (2017).

[35] E. Constant, V. D. Taranukhin, A. Stolow, and P. B. Corkum, Methods for the measurement of the duration of high-harmonic pulses, Phys. Rev. A. 56, 3870 (1997).

[36] P. Eckle, M. Smolarski, P. Schlup, J. Biegert, A. Staudte, M. Schöffler, H. G. Muller, R. Dörner, and U. Keller, Attosecond angular streaking, Nat. Phys. 4, 565 (2008).

[37] C. Pellegrini, A. Marinelli, and S. Reiche, The physics of x-ray free-electron lasers, Rev. Mod. Phys. 88, 015006 (2016).

[38] E. L. Saldin, E. A. Schneidmiller, and M. V. Yurkov, Statistical and coherence properties of radiation from x-ray free-electron lasers, New J. Phys. 12, 35010 (2010).

[39] S. Krinsky and R. L. Gluckstern, Analysis of statistical correlations and intensity spiking in the self-amplified spontaneousemission free-electron laser, PRAB 6, 050701 (2003).

[40] J. Itatani, F. Quéré, G. L. Yudin, M. Y. Ivanov, F. Krausz, and P. B. Corkum, Attosecond Streak Camera, Phys. Rev. Lett. 88, 173903 (2002).

[41] N. Hartmann, W. Helml, A. Galler, M. R. Bionta, J. Grünert, S. L. Molodtsov, K. R. Ferguson, S. Schorb, M. L. Swiggers, S. Carron, C. Bostedt, J.-C. Castagna, J. Bozek, J. M. Glownia, D. J. Kane, A. R. Fry, W. E. White, C. P. Hauri, T. Feurer, and R. N. Coffee, Sub-femtosecond precision measurement of relative $\mathrm{x}$-ray arrival time for free-electron lasers, Nat. Photon. 8, 706 (2014).

[42] M. R. Bionta, N. Hartmann, M. Weaver, D. French, D. J. Nicholson, J. P. Cryan, J. M. Glownia, K. Baker, C. Bostedt, M. Chollet, Y. Ding, D. M. Fritz, A. R. Fry, D. J. Kane, 
J. Krzywinski, H. T. Lemke, M. Messerschmidt, S. Schorb, D. Zhu, W. E. White, and R. N. Coffee, Spectral encoding method for measuring the relative arrival time between $\mathrm{x}$-ray/optical pulses, Rev. Sci. Instrum. 85, 83116 (2014).

[43] C. Gahl, A. Azima, M. Beye, M. Deppe, K. Döbrich, U. Hasslinger, F. Hennies, A. Melnikov, M. Nagasono, A. Pietzsch, M. Wolf, W. Wurth, and A. Föhlisch, A femtosecond X-ray/optical cross-correlator, Nat. Photon. 2, 165 (2008).

[44] P. Emma, K. Bane, M. Cornacchia, Z. Huang, H. Schlarb, G. Stupakov, and D. Walz, Femtosecond and Subfemtosecond Xray Pulses from a Self-Amplified Spontaneous-Emission-Based Free-Electron Laser, Phys. Rev. Lett. 92, 074801 (2004).

[45] M. O. Krause, F. A. Stevie, L. J. Lewis, T. A. Carlson, and W. E. Moddeman, Multiple excitation of neon by photon and electron impact, Phys. Lett. A 31, 81 (1970).

[46] E. Allaria, B. Diviacco, C. Callegari, P. Finetti, B. Mahieu, J. Viefhaus, M. Zangrando, G. de Ninno, G. Lambert, E. Ferrari, J. Buck, M. Ilchen, B. Vodungbo, N. Mahne, C. Svetina, C. Spezzani, S. Di Mitri, G. Penco, M. Trovó, W. M. Fawley, P. R. Rebernik, D. Gauthier, C. Grazioli, M. Coreno, B. Ressel, A. Kivimäki, T. Mazza, L. Glaser, F. Scholz, J. Seltmann, P. Gessler, J. Grünert, A. de Fanis, M. Meyer, A. Knie, S. P. Moeller, L. Raimondi, F. Capotondi, E. Pedersoli, O. Plekan, M. B. Danailov, A. Demidovich, I. Nikolov, A. Abrami,
J. Gautier, J. Lüning, P. Zeitoun, and L. Giannessi, Control of the Polarization of a Vacuum-Ultraviolet, High-Gain, FreeElectron Laser, Phys. Rev. X 4, 041040 (2014).

[47] A. A. Lutman, T. J. Maxwell, J. P. MacArthur, M. W. Guetg, N. Berrah, R. N. Coffee, Y. Ding, Z. Huang, A. Marinelli, S. Moeller, and J. C. U. Zemella, Fresh-slice multicolour x-ray free-electron lasers, Nat. Photon. 10, 745 (2016).

[48] J. Gagnon and V. S. Yakovlev, The direct evaluation of attosecond chirp from a streaking measurement, Appl. Phys. B 103, 303 (2011).

[49] Y. Ding, F.-J. Decker, P. Emma, C. Feng, C. Field, J. Frisch, Z. Huang, J. Krzywinski, H. Loos, J. Welch, J. Wu, and F. Zhou, Femtosecond X-ray Pulse Characterization in Free-Electron Lasers Using a Cross-Correlation Technique, Phys. Rev. Lett. 109, 254802 (2012).

[50] M. C. Hoffmann, I. Grguraš, C. Behrens, C. Bostedt, J. Bozek, H. Bromberger, R. Coffee, J. T. Costello, L. F. Dimauro, Y. Ding, G. Doumy, W. Helml, M. Ilchen, R. Kienberger, S. Lee, A. R. Maier, T. Mazza, M. Meyer, M. Messerschmidt, S. Schorb, W. Schweinberger, K. Zhang, and A. L. Cavalieri, Femtosecond profiling of shaped x-ray pulses, New J. Phys. 20, 33008 (2018).

[51] V. Schmidt, Photoionization of atoms using synchrotron radiation, Rep. Prog. Phys. 55, 1483 (1992). 